\title{
Review Article \\ Geometric Models for Isotropic Random Porous Media: A Review
}

\author{
Helmut Hermann ${ }^{1}$ and Antje Elsner ${ }^{2}$ \\ ${ }^{1}$ Institute for Solid State and Materials Research, IFW Dresden, P.O. Box 270116, 01171 Dresden, Germany
}

${ }^{2}$ Institute for Informatics, TU Dresden, 01062 Dresden, Germany

Correspondence should be addressed to Helmut Hermann; h.hermann@ifw-dresden.de

Received 9 October 2013; Accepted 26 February 2014; Published 30 April 2014

Academic Editor: Thomas Hipke

Copyright (c) $2014 \mathrm{H}$. Hermann and A. Elsner. This is an open access article distributed under the Creative Commons Attribution License, which permits unrestricted use, distribution, and reproduction in any medium, provided the original work is properly cited.

\begin{abstract}
Models for random porous media are considered. The models are isotropic both from the local and the macroscopic point of view; that is, the pores have spherical shape or their surface shows piecewise spherical curvature, and there is no macroscopic gradient of any geometrical feature. Both closed-pore and open-pore systems are discussed. The Poisson grain model, the model of hard spheres packing, and the penetrable sphere model are used; variable size distribution of the pores is included. A parameter is introduced which controls the degree of open-porosity. Besides systems built up by a single solid phase, models for porous media with the internal surface coated by a second phase are treated. Volume fraction, surface area, and correlation functions are given explicitly where applicable; otherwise numerical methods for determination are described. Effective medium theory is applied to calculate physical properties for the models such as isotropic elastic moduli, thermal and electrical conductivity, and static dielectric constant. The methods presented are exemplified by applications: small-angle scattering of systems showing fractal-like behavior in limited ranges of linear dimension, optimization of nanoporous insulating materials, and improvement of properties of open-pore systems by atomic layer deposition of a second phase on the internal surface.
\end{abstract}

\section{Introduction}

The development of models for random porous media and the setting-up of structure-property relationships have benefitted substantially from the rich body of theoretical work on disordered matter. Beginning with Ziman's famous book on models of disorder [1] treatises like that on the physics of structurally disordered matter [2], the physics of foam [3], mechanical properties of heterogeneous materials [4], effective medium theory for disordered microstructures [5], structure-property relations of random heterogeneous materials [6], transport, and flow in porous media [7] followed. Parallel to this progress in physics, mathematical methods for the description of random systems have been improved as documented in textbooks on stochastic geometry and its applications [8-10]. Activities of bridging ideas developed in physics and materials science on the one hand and of methods established by mathematicians on the other hand can be found, for example, in [10-14].
In this review we consider models for random isotropic porous media of both closed-pore and open-pore type. The pore surfaces have, at least piecewise, spherical curvature with arbitrary distribution of radii of curvature (for simplicity, called pore size distribution below). There is no restriction regarding the volume fraction of pores. The considered models are based on rules for arranging spheres in the threedimensional space. Three types of rules are used: random arrangement of noninteracting spheres (Boolean model, this model is not restricted to spherical shape of pores), denserandom packing of hard spheres (DRP), and random packing of partially penetrable spheres each consisting of a hard core and a soft shell (cherry-pit model). The latter one appears as a numerical interpolation scheme between the Boolean model and the DRP method. A model for a porous medium is made by arranging spheres according to one of the above rules and, thereafter, allotting solid material to the space of the model which is not covered by any sphere. Such a model can be refined by coating the pore surfaces with a second solid phase. 
The models are characterized by their basic geometrical features-volume fraction of pores, $V_{V}$, specific surface area, $S_{V}$, pore size distribution, $f(r)$, and correlation function, $C(r)$. On one hand, these characteristics are required to interpret experimental data, for example, small-angle scattering curves. On the other hand, they are important for understanding and control of the interplay between structure and physical properties of random porous media.

Physical properties of the models are discussed in terms of an effective medium approach. Explicit expressions for macroscopic elastic moduli, thermal and electrical conductivity, and static dielectric constant are given.

The definitions of the geometrical characteristics are explained in Section 2, the models are described in Section 3, physical properties are discussed in Section 4, and applications to experimental situations are presented in Section 5.

\section{Geometrical Characteristics}

The pore space is described as a random set $A$. We consider volume fraction $V_{V}(A)$, specific surface area $S_{V}(A)$, pore size distribution $f(r)$, and correlation function $C_{A}(r)$ as geometrical characteristics of $A$. It is useful to introduce the complement of $A$ consisting of the part of the space which does not belong to $A$. The complement of $A$ is indicated by $A^{c}$. This notation is standard in stochastic geometry [10]. For example, if $A$ denotes the pore space of the porous medium, $c_{p}=V_{V}(A)$ and $c_{b}=V_{V}\left(A^{c}\right)=1-c_{p}$ are the volume fraction of the pore space and the solid bulk phase, respectively. $S_{V}(A)=S_{V}\left(A^{c}\right)$ is the same for $A$ and $A^{c}$.

The pore space $A$ is described by the shape function:

$$
s^{p}(\mathbf{r})= \begin{cases}1, & \mathbf{r} \in A \\ 0, & \text { otherwise. }\end{cases}
$$

The volume fraction $c_{p}$ of pores is defined by the probability of finding a random test point $\mathbf{r}_{1}$ in $A$ :

$$
c_{p}=V_{V}(A)=P\left(\mathbf{r}_{1} \in A\right) .
$$

If $s^{p}(\mathbf{r})$ is known $c_{p}$ can be calculated by

$$
c_{p}=\frac{1}{V} \int_{V} s^{p}(\mathbf{r}) d V .
$$

The correlation function

$$
C_{p}(\mathbf{r})=P\left(\mathbf{r}_{1} \in A, \mathbf{r}_{2} \in A\right), \quad \mathbf{r}=\mathbf{r}_{1}-\mathbf{r}_{2},
$$

denotes the probability of finding two random test points $\left(\mathbf{r}_{1}, \mathbf{r}_{2}\right)$ inside the pore space. It is calculated from the shape function by

$$
C_{p}(\mathbf{r})=\frac{1}{V} \int_{V} s^{p}(\mathbf{u}+\mathbf{r}) s^{p}(\mathbf{u}) d V_{\mathbf{u}}
$$

Because of isotropy it depends only on the distance $r=\mid \mathbf{r}_{1}-$ $\mathbf{r}_{2} \mid$ between the test points

$$
C_{p}(r)=\frac{1}{4 \pi} \int_{\Omega} C_{p}(\mathbf{r}) d \Omega
$$

where $\Omega$ is the solid angle. Replacing index $p$ by $b$ and setting $s^{b}(\mathbf{r})=1-s^{p}(\mathbf{r})$ one obtains from $((1),(5)$, and (6)) for the correlation function of the solid phase

$$
C_{b}(r)=1-2 c_{p}+C_{p}(r)
$$

The specific surface area of $A$ is defined by

$$
s_{p}=S_{V}(A)=\lim _{\epsilon \rightarrow 0} \frac{P\left(\mathbf{r}_{1} \in S_{\epsilon}\right)}{2 \epsilon},
$$

where $P\left(\mathbf{r}_{1} \in S_{\epsilon}\right)$ is the probability of finding a random test point $\mathbf{r}_{1}$ in $S_{\epsilon}$ and $S_{\epsilon}$ is defined by all points of the threedimensional space the distance of which from the surface $S(A)$ is less than $\epsilon$. If the correlation function of the system is known, $s_{p}$ is related to $C_{p}(r)$ by $[10]$

$$
s_{p}=-4 \lim _{r \rightarrow 0} \frac{d C_{p}(r)}{d r} .
$$

For illustration we consider the most simple case of a single pore with radius $R$ in a sample of volume $V$ as a model for a dilute system of equal pores; that is, $c_{p} \ll 1$. The volume fraction and the specific surface area are given by $c_{p}=4 / 3 \pi R^{3} / V$ and $s_{p}=4 \pi R^{2} / V$, respectively. The integral in formula (5) is evaluated as the convolution integral of a spherical shape function [16]. Neglecting terms of the order $c_{p}^{2}$ one obtains

$$
C_{p}(r)= \begin{cases}c_{p}\left(1-\frac{3 r}{4 R}+\frac{r^{3}}{16 R^{3}}\right), & 0 \leq r \leq 2 R \\ 0, & r>2 R .\end{cases}
$$

Polydisperse systems are additionally characterized by a size distribution function $f(r)$ with mean value

$$
m=\int x f(x) d x
$$

and variance

$$
\sigma^{2}=\int(x-m)^{2} f(x) d x
$$

\section{Models}

3.1. Poisson Grain Model. The Poisson grain model (also known as Boolean model, see [10]) is an important and versatile model for generating random two-phase structures. It is created in two steps. In the first step a random (Poisson) point field is generated (here in the three-dimensional space) with number density $v$ of points. This point field has the following property. Considering an arbitrary finite region $B$ with volume $V(B)$, the number $N(B)$ of points situated within $B$ is a random variable. The probability that $N(B)$ takes the value $n$ is

$$
P(N(B)=n)=\frac{[\nu V(B)]^{n}}{n !} \exp (-\nu V(B)), \quad n=0,1, \ldots
$$


The numbers $N\left(B_{1}\right), N\left(B_{2}\right), \ldots$ are independent random variables if the regions $B_{1}, B_{2}, \ldots$ do not overlap. Practically, the point field, that is, the coordinates of the points, can be produced using a random number generator for numbers equally distributed in finite coordinate intervals.

In the second step, respectively, one sphere is placed on each point of the point field where the size distribution of the spheres is arbitrary. The set-theoretical union of all spheres represents a random set $A$ with volume fraction

$$
V_{V}(A)=1-\exp (-\nu \bar{V}),
$$

where $\bar{V}=(4 \pi / 3) \int_{0}^{\infty} x^{3} f(x) d x$ is the mean volume of the spheres.

Consider definition (2) of volume fraction for the special case that $A$ is generated using equal spheres with radius $R$ and choose a sphere of the same radius for $B$ in (13). Then the probability that $B$ does not contain any point of the Poisson point field, that is, $n=0$ in (13), is equal to the probability that the center of the sphere $B$ has always a distance $r>R$ to any point of the point field. This is exactly definition (2) for the volume fraction of $A^{c}, V_{V}\left(A^{c}\right)=\exp \left(-\nu(4 \pi / 3) r^{3}\right)=$ $1-V_{V}(A)$ which gives (14) for the considered special case. The general result (14) is obtained using the same idea.

The specific surface area of the model is

$$
S_{V}=\nu\left[1-V_{V}(A)\right] \bar{S}
$$

where $\bar{S}=4 \pi \int_{0}^{\infty} x^{2} f(x) d x$ is the mean surface area of the spheres.

The correlation function as defined in (5) and (6) is

$$
C_{A}(r)=2 c_{A}-1+\left(1-c_{A}\right)^{2} \exp (\nu \gamma(r)),
$$

where $c_{A}=V_{V}(A)$ and $\gamma(r)$ is the correlation function of a single sphere averaged over the size distribution $f(r)$ according to

$$
\gamma(r)=\frac{4 \pi}{3} \int_{r / 2}^{\infty} x^{3}\left(1-\frac{3 r}{4 x}+\frac{r^{3}}{16 x^{3}}\right) f(x) d x .
$$

The first formulation of the model was given by Porod [17] for equal spheres. A fundamental and general description can be found in [10].

Identifying the space covered by spheres in the Boolean model with the pore space of a random porous medium, one obtains

$$
\begin{gathered}
c_{p}=V_{V}(A), \\
s_{p}=S_{V} .
\end{gathered}
$$

Figure 1 shows a cutout of a Boolean model with constant distribution of radii in the interval from 1 to 2 . Note that some of the small spheres may be not visible because they can be covered completely by large spheres.

3.2. Random Packing of Hard Spheres. The model of hard spheres is easily defined by the condition that two particles

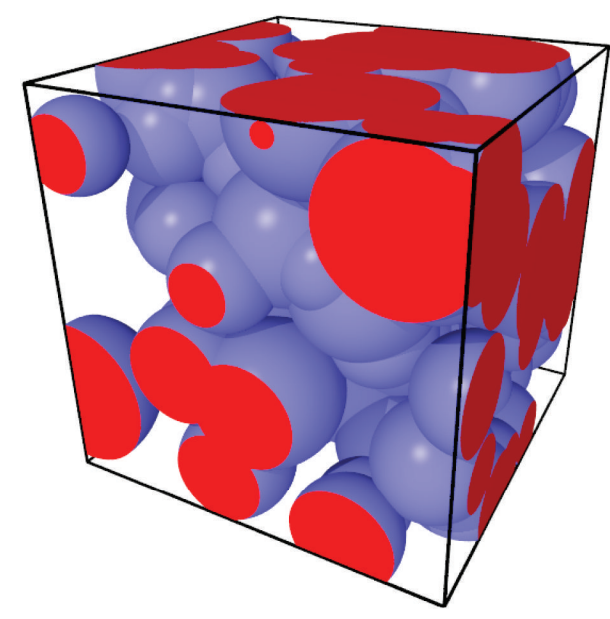

FIGURE 1: Cutout of a Boolean model with sphere radii equally distributed in the interval $(1,2)$, box size $=5$, and volume fraction $V_{V}=0.81$.

cannot overlap. Despite the simplicity of the model it appears very complex especially if the packing fraction of spheres is high. For equal spheres, packing fractions of 0.74 (regular hexagonal and face-centered cubic structure), 0.68 (regular body-centered cubic), and 0.636 (dense-random packing) are obtained. While regular packing of spheres has been known as a model for the arrangement of atoms in simple crystal structures [18], Bernal [19] proposed the model of dense-random packed spheres as an approach to the structure of simple liquids. Early reviews on construction algorithms and applications of randomly packed sphere models can be found in [20-22]. More recently, Löwen [23] reviewed methods for analyzing hard sphere systems such as the Percus-Yevick theory, the density-functional approach, and methods of computer simulations from the physical point of view. Mathematical theories are described in [10].

In this work we use the force-biased algorithm for the generation of computer models of hard spheres. This algorithm includes the simulation of systems with arbitrary size distribution of spheres and works very efficiently [2426]. The algorithm starts with a dilute system of randomly distributed spheres in a parallelepipedal container with periodic boundary conditions applied. Initially, each sphere $i$ has an inner hard core diameter $2 r_{i}^{h, 0}$ and an outer diameter $2 r_{i}^{s, 0}$. During iteration step $n$, distances $r_{i j}<r_{i}^{h, n}+r_{j}^{h, n}$ between two spheres are not allowed. No interaction occurs for $r_{i j} \geq$ $r_{i}^{s, n}+r_{j}^{s, n}$. If $r_{i}^{h, n}+r_{j}^{h, n}<r_{i j}<r_{i}^{s, n}+r_{j}^{s, n}$, sphere $i$ is shifted from position $\mathbf{x}_{i}^{n}$ to position $\mathbf{x}_{i}^{n+1}$ according to

$$
\mathbf{x}_{i}^{n+1}=\mathbf{x}_{i}^{n}+\sum_{j=1}^{z_{i}} P_{i j} \frac{\mathbf{x}_{i}^{n}-\mathbf{x}_{j}^{n}}{\left|\mathbf{x}_{i}^{n}-\mathbf{x}_{j}^{n}\right|},
$$

where $P_{i j}$ depends on the diameters of spheres $i$ and $j$ and on control parameters and $z_{i}$ is the number spheres which have overlapped with sphere $i$. Quantity $P_{i j}$ is only used to optimize the convergence of the algorithm. It does not represent any physical interaction. After each step $n$ the packing fraction is 


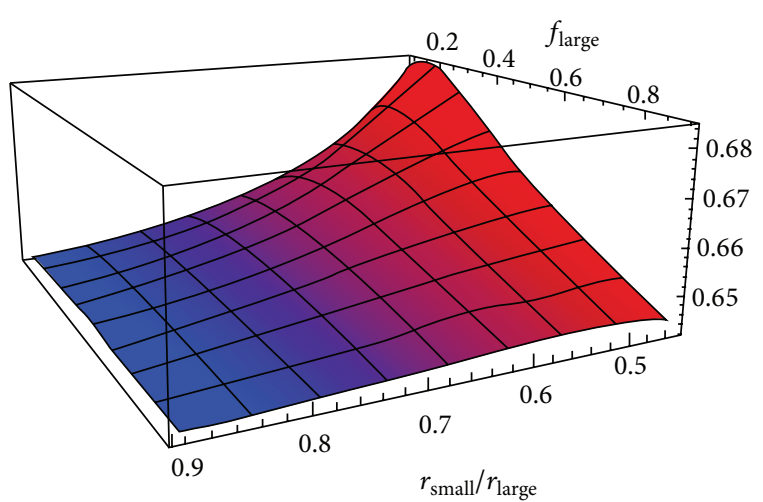

Figure 2: Volume fraction of systems of dense-random packed spheres with bimodal distribution of radii versus size ratio $r_{\text {small }} / r_{\text {large }}$ of spheres and number fraction $f_{\text {large }}$ of large spheres.

enhanced by adjusting the sphere diameters at constant box size. Denoting the maximum number of iteration steps by $n_{\max }, r_{i}^{s, n}-r_{i}^{h, n} \rightarrow 0$ for all $i$ if $n \rightarrow n_{\max }$.

The algorithm is terminated if either the desired packing fraction is attained, or if no further progress can be achieved, or if $n=n_{\max }$. For details see [24-27].

The volume fraction of spheres and the specific surface area of a system of packed hard spheres with number density $\nu=N / V$ are simply given by

$$
\begin{aligned}
& V_{V}=v \int_{0}^{\infty} \frac{4 \pi}{3} r^{3} f(r) d r \\
& S_{V}=v \int_{0}^{\infty} 4 \pi r^{3} f(r) d r
\end{aligned}
$$

respectively.

Figure 2 shows $V_{V}$ of systems of dense-random packed hard spheres with bimodal size distribution. For a size ratio of 0.45 of small-to-large spheres the volume fraction has a maximum of 0.684 at a number fraction of 0.2 of large spheres. The maximum packing fraction decreases as the size ratio approaches 1 and takes the value of 0.636 for equal spheres.

There are no exact formulas for the correlation function of dense hard sphere systems. Current approximations and discussions of this problem can be found, for example, in $[10,11,23]$. Monte-Carlo simulations based on the definition (4) can be used to calculate the correlation function for numerically simulated hard sphere systems.

3.3. Penetrable Sphere Models. Penetrable sphere models can be used to describe open-pore structures. Widom and Rowlinson [28, 29] introduced a penetrable sphere model for the study of liquid-vapor phase transitions. In this model, the spheres are allowed to overlap completely. The main difference to the Boolean model described above consists in the definition of a potential energy which is related to the volume occupied by the overlapping spheres. The WidomRowlinson model was first extended and applied to studies on phase behavior of complex fluids in [30, 31]. Similar models were described by a number of authors [32-40]. Note that the force-biased algorithm described in the previous

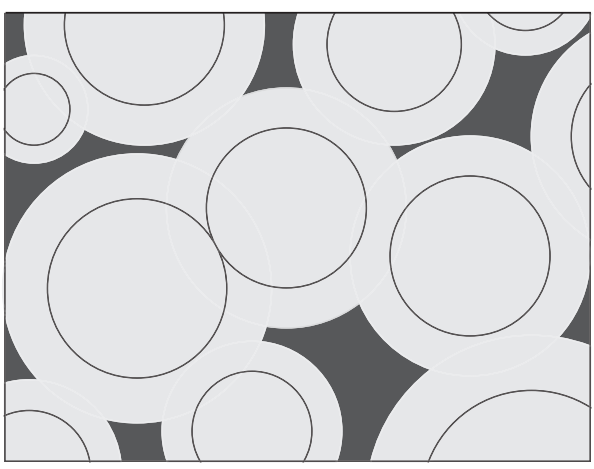

FIgure 3: Construction scheme of the cherry-pit model. Circles (solid lines) mark the pits, gray regions are the spheres which partially overlap, and the solid material is black.

section shows features of a penetrable sphere model during the rearrangement process.

Torquato [41-43] introduced the penetrable concentricshell model which is called cherry-pit model for brevity. In this model, each sphere of radius $R$ is composed of an impenetrable core of radius $\lambda R$ (the pit) surrounded by a perfectly penetrable shell of thickness $(1-\lambda) R$. For the case of randomly distributed sphere centers, the limits of $\lambda=0$ and $\lambda=1$ correspond to the Boolean model and the random hard sphere one, respectively. Approximate analytical expressions for volume fraction and specific surface area of this model were presented in $[6,39,40,44]$. Results of numerical simulations with equally sized spheres [45] agreed reasonably with the predictions for the volume fractions by $[39,40]$. Formulas with improved accuracy for volume fraction and specific surface area of the cherry-pit model with equally sized spheres have been given in [46, 47]. The cherry-pit model is a variable model which appears as an interpolation scheme communicating between the Boolean model and the hard sphere packing paradigm. Also it allows to vary the degree of open-porosity by changing the value of $\lambda$. While previous studies of the cherry-pit model concentrated on using equally sized spheres actual progress has been achieved by introducing arbitrary size distributions of the spheres [48].

Samples of the cherry-pit model can be generated by means of the force-biased procedure for packing of hard spheres. After choosing the number $N$ of the spheres, the desired size distribution, and the intended packing fraction, the procedure is started. The result consists in the set of the coordinates $\left(x_{i}, y_{i}, z_{i}\right), i=1,2,3, \ldots, N$, the radii $\lambda r_{i}$ of the spheres, and the achieved packing fraction $\phi_{h}$ given by (20). The corresponding cherry-pit model is obtained by identifying the spheres of the simulated system with the pits of the cherry-pit model. The union of all spheres with radii $r_{i}$ represents the pore space of the model. The procedure is illustrated in Figure 3. Figure 4 shows cherrypit models generated from a system of randomly packed pits with Gamma distributed radii

$$
f(r)=\frac{b^{p}}{\Gamma(p)} r^{p-1} \exp (-b r), \quad r \geq 0,
$$

and $\bar{r}=p / b, \sigma^{2}=p / b^{2}, \Gamma(p)=\int_{0}^{\infty} t^{p-1} \exp (-t) d t$. 


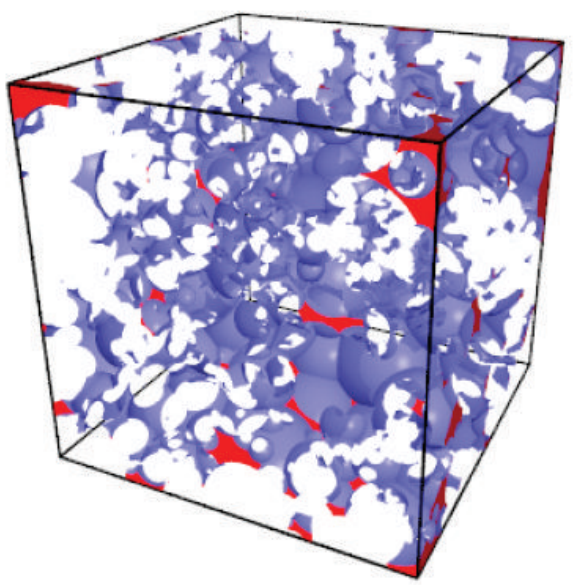

(a)

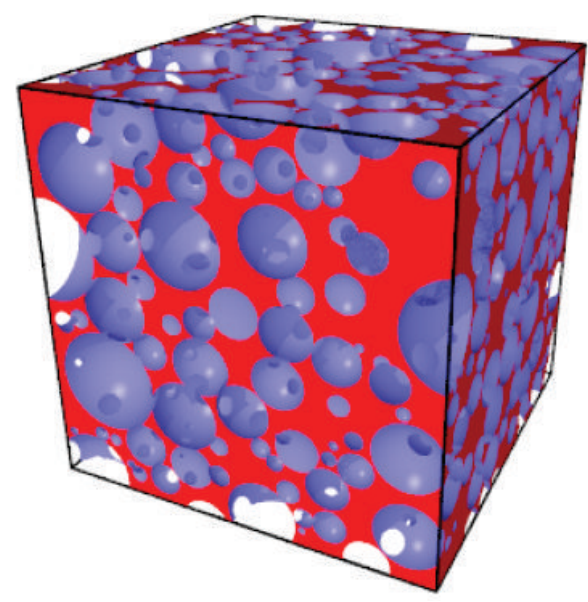

(c)

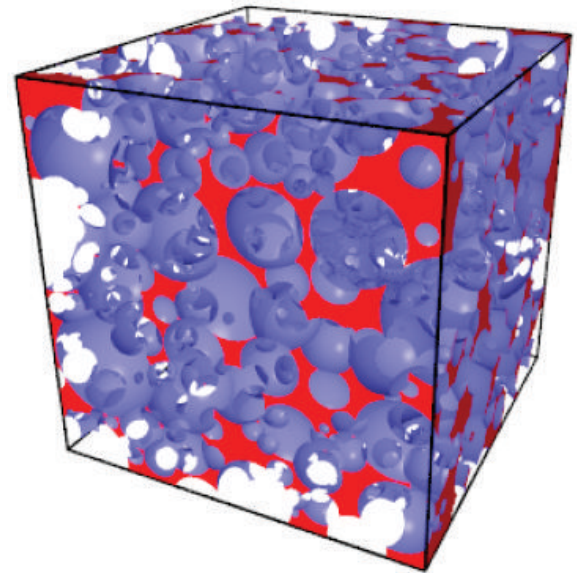

(b)

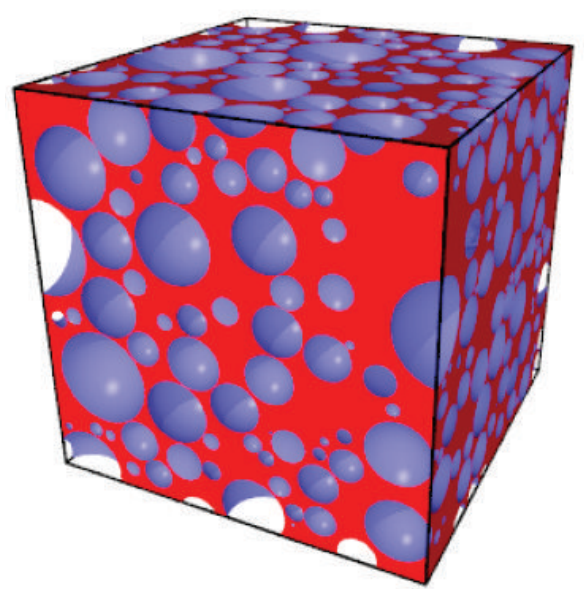

(d)

FIGURE 4: Models for porous media generated using the cherry-pit model; Gamma distributed pore size; mean pore size $\bar{r}=1$, and variance $\sigma^{2}=0.5$; packing fraction of the pit system $\phi_{h}=0.60 ; \lambda=0.75,0.85,0.95,1.00$ for ((a), (b), (c), and (d)).

The samples shown in Figure 4 differ by the values of $\lambda$ chosen for the construction of the cherry-pit models. Clearly, the degree of open-porosity rises with decreasing $\lambda$-values.

The volume fraction of a simulated model is determined using the point-count method where random test points are scattered in the simulation box. The number of test points situated in pores divided by the number of all test points gives an estimate for the volume fraction of pores. The specific surface area is calculated in a similar way. Here, the random test points are distributed on the surfaces of the spheres of the cherry-pit model. The number of points on the surface of a sphere not covered by any other sphere contributes to the surface of the model. The number of contributing points to the number of all points gives an estimate for the surface area of the model. Figure 5 illustrates the statistical reliability of the simulations and its dependence on the size of the model measured by the number of spheres of the simulated cherrypit model. The standard deviation of both $V_{V}$ and $S_{V}$, each divided by the mean value of the corresponding quantity, decreases with increasing box size. The statistical accuracy of the volume fraction is of the order of $0.25 \%$ for $N \geq 10^{4}$. The specific surface area is determined with an statistical accuracy of less than $2 \%$ for $N=10^{4}$ and $0.5 \%$ for $N=10^{5}$.

Statistical analysis of large numbers of simulated monodisperse [47] and polydisperse [48] cherry-pit models revealed that the volume fraction, $V_{V}$, of spheres and the specific surface area, $S_{V}$, are given by the approximate formulas:

$$
\begin{gathered}
V_{V}=\phi_{h}+\left(1-\phi_{h}\right) \Phi\left(\frac{3 \sqrt{2 \pi}}{2} \frac{(1-\lambda) \phi_{h}}{\lambda\left(1-\phi_{h}\right)}\right), \\
\Phi(z)=\frac{2}{\sqrt{\pi}} \int_{0}^{z} \exp \left(-t^{2}\right) d t, \\
S_{V}=\frac{3}{\lambda r_{\text {ref }}} \phi_{h} \exp \left(-\frac{9 \pi}{4} \frac{(1-\lambda)^{2} \phi_{h}^{2}}{\lambda^{2}\left(1-\phi_{h}\right)^{2}}\right) .
\end{gathered}
$$

Again, relations (18) apply if the spheres describe the pore space of a medium. Expressions (23) and (25) depend on the quantities $\phi_{h}$ and $\lambda$, which characterize the basic system of randomly packed hard spheres and the construction scheme 


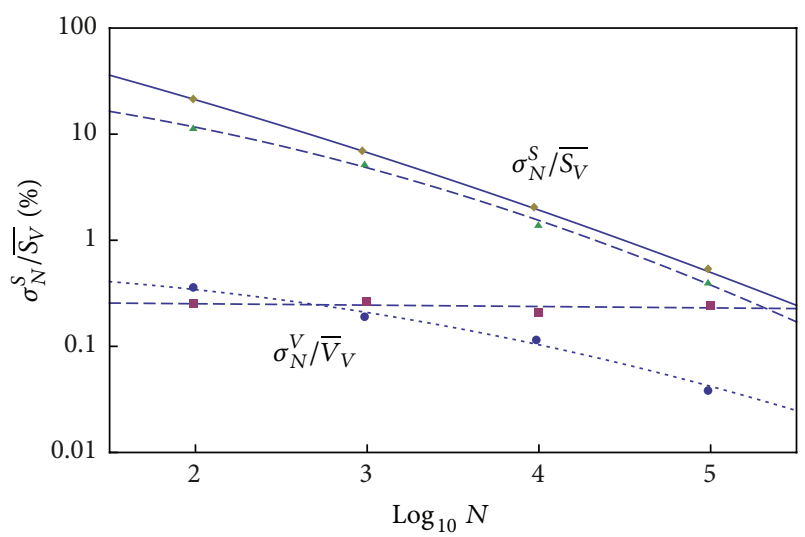

Figure 5: Normalized standard deviation $\sigma_{N}^{V} / \bar{V}_{V}$ and $\sigma_{N}^{S} / \bar{S}_{V}$ of respectively volume fraction, $V_{V}$, and specific surface area, $S_{V}$, of cherry-pit models with Gamma distributed radii and $\sigma / \bar{r}=0.707$ versus number $N$ of spheres used for the construction of a model. The standard deviation is calculated from 10 samples for each model. $S_{V}$ : rhombuses and solid line: $\lambda=0.75$, triangles and dashed line: $\lambda=0.95 ; V_{V}$ : circles and dotted line: $\lambda=0.75$, squares and dashed line: $\lambda=0.95$.

of the cherry-pit model, respectively. Parameter $r_{\text {ref }}$ defines the unit of length of the system. For monodisperse systems it is equal to the sphere radius. In the polydisperse case the relation

$$
\frac{r_{\mathrm{ref}}}{\bar{r}}=a+b\left(\frac{\sigma}{\bar{r}}\right)^{2}
$$

with $a=1.014 \pm 0.002, b=0.454 \pm 0.006$ follows from fitting (25) to the values of the specific surface areas of simulated cherry-pit models [48]. Parameters $\bar{r}$ and $\sigma$ are the mean value and the standard deviation of the radii distribution, respectively, defined by ((11), (12)). The error appearing for $\sigma \rightarrow 0$ in (26) is below $2 \%$.

Expression (23) does not depend on any parameter related to the size distribution of spheres. So it appears as quite a general relation. Detailed analyses in [48] showed that (23) is most reliable for systems with $0.40 \leq \phi_{h} \leq 0.71, c_{p} \leq$ 0.8 and for size distributions with $\sigma / \bar{r}$ up to about $3.5(\bar{r}$ is the mean radius of spheres). Figure 6 compares (23) to simulated data obtained from systems with 10.000 spheres generated at periodic boundary conditions where the radii are distributed according to a Gamma distribution [48].

Formula (25) is accurate in the limits of few percent for $\sigma \leq 0.25$. The impact of the skewness of the size distributions on the accuracy of (25) is negligible. Figure 7 illustrates the validity of (25).

\subsection{Interphase Models}

3.4.1. Limit of Very Thin Interphase Layer. We start with one of the models for a porous medium with a single bulk phase. The pore space is characterized by its volume fraction $V_{V}$ and the specific surface area $S_{V}$. Then, the internal surface

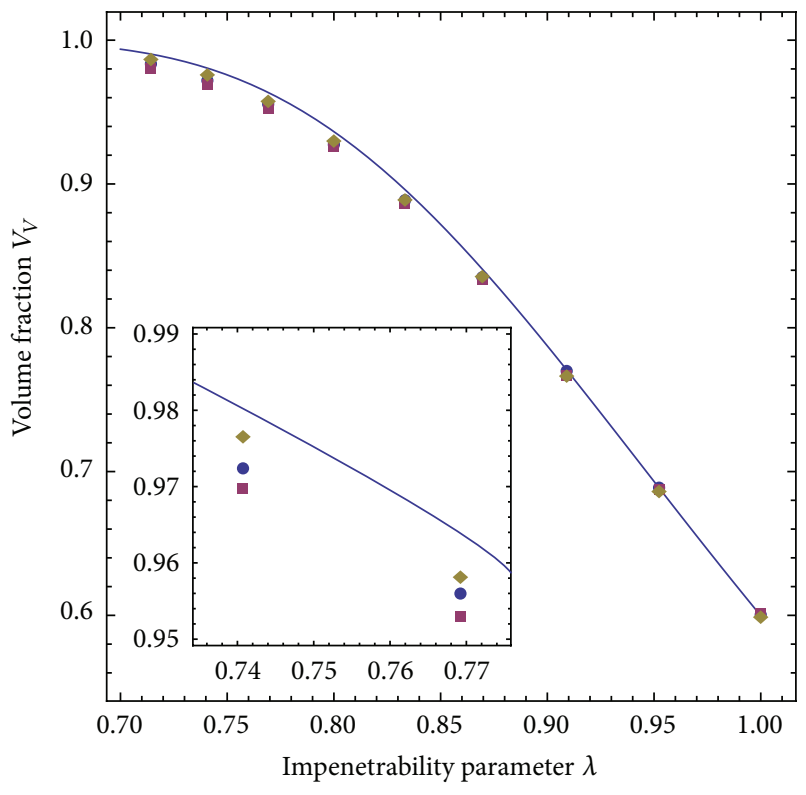

FIgURE 6: Volume fraction of cherry-pit models for Gamma distributed pore size with $\sigma / \bar{r}=0.25$ (rhombs), 0.71 (squares), and 1.00 (circles).

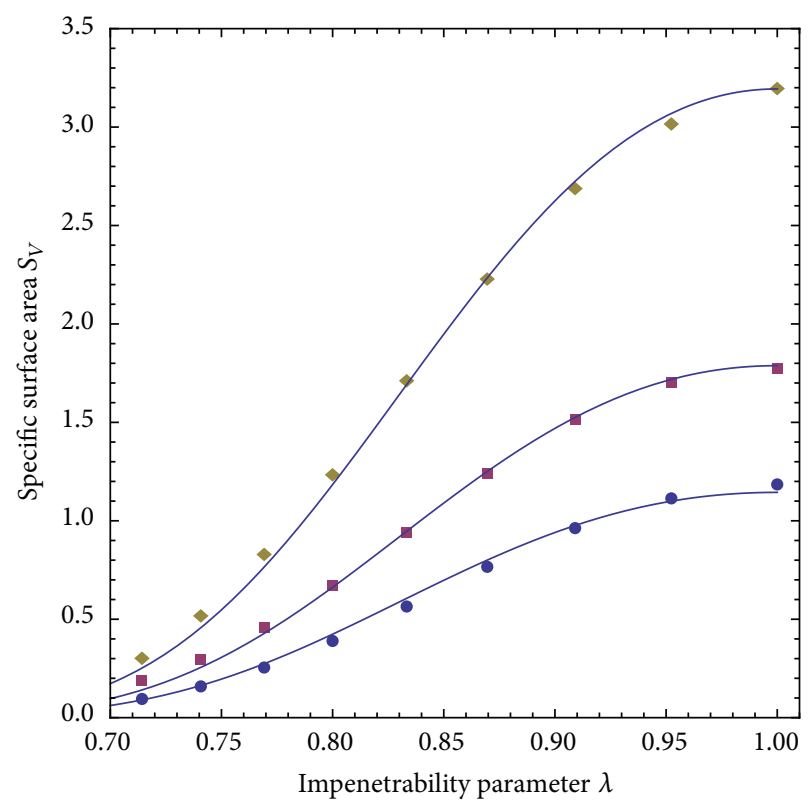

FIGURE 7: Specific surface area of cherry-pit models for Gamma distributed pore size with $\sigma / \bar{r}=0.25$ (rhombs), 0.71 (squares), and 1.00 (circles).

of the model is coated by a layer of a second solid phase of thickness $\delta t$. This layer is called interphase. Denoting the smallest radius of curvature of a pore of the model by $r_{\min }$, for $\delta t \ll r_{\min }$, the volume fraction of the layer is approximately

$$
c_{i}=\delta t S_{V},
$$

the volume fraction of the pores reduces to

$$
c_{p}=V_{V}-\delta t S_{V}
$$




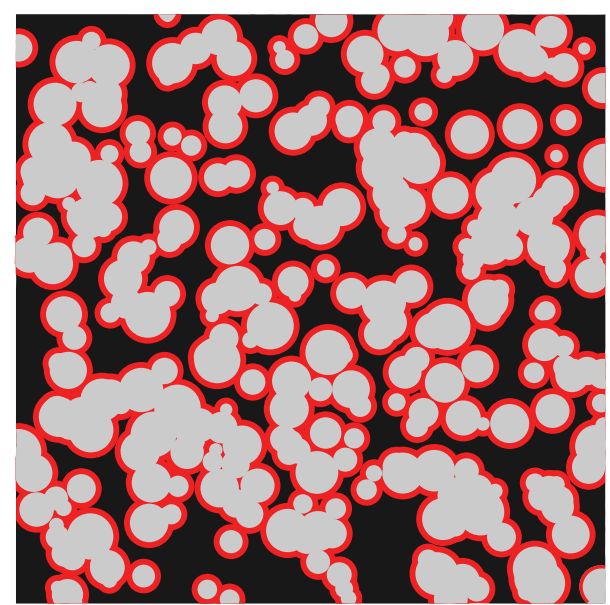

FIGURE 8: Planar intersection of a Boolean interphase model, poreslight gray, bulk phase-black, and interphase layer-red.

and the volume fraction of the bulk phase remains

$$
c_{b}=1-V_{V}=1-c_{p}-c_{i} .
$$

3.4.2. Boolean Interphase Model. Another way to calculate the volume fraction of the interphase is to compare two models differing only by the size of the spheres used for the construction. Consider a Boolean model 1 generated using spheres with size distributions $f_{1}(r)$. The space not covered by any sphere defines the bulk phase 1 . Reducing the radius of each of the spheres of exactly this model by $\delta t$ a second model 2 is obtained with size distribution $f_{2}(r)=f_{1}(r-\delta t)$, where $f_{1}(r)=0$ for $r \leq \delta t$ is required. This model defines the pore space. Then, the interphase of thickness $\delta t$ is given by the space neither belonging to the bulk phase 1 nor to the pore space and the volume fraction of the interphase follows as

$$
c_{i}=V_{V}(1)-V_{V}(2) .
$$

Figure 8 shows the intersection of a Boolean interphase model. For the special case of Boolean models with spheres of equal radius $R$ one obtains

$$
c_{i}=\left\{1-\exp \left(-v \frac{4 \pi}{3} R^{3}\right)\right\}-\left\{1-\exp \left(-v \frac{4 \pi}{3}(R-\delta t)^{3}\right)\right\} .
$$

For $\delta t \ll R$,

$$
c_{i}=\exp \left(-\nu \frac{4 \pi}{3} R^{3}\right) 4 \pi \nu R^{2} \delta t=\nu\left(1-V_{V}(1)\right) 4 \pi R^{2} \delta t
$$

follows which corresponds to ((15), (27)).

3.4.3. Hard Sphere Interphase Models. For interphase models based one hard sphere systems the volume fraction of the interphase is calculated similarly. Assuming a Gaussian distribution of radii

$$
f(r)=\frac{1}{\sqrt{2 \pi} \sigma} \exp \left(-\frac{(r-\bar{r})^{2}}{2 \sigma^{2}}\right)
$$

the volume fraction of the interphase is according to (20):

$$
c_{i}=\frac{4 \pi \rho}{3}\left\{\bar{r}\left[\bar{r}^{2}+3 \sigma^{2}\right]-(\bar{r}-\delta t)\left[(\bar{r}-\delta t)^{2}+3 \sigma^{2}\right]\right\} .
$$

In the limit $\delta t \ll \bar{r}$ one obtains with ((21), (33)) analogously to (27)

$$
c_{i}=4 \pi \rho\left(\bar{r}^{2}+\sigma^{2}\right) \delta t=S_{V} \delta t
$$

3.4.4. Cherry-Pit Interphase Model. The cherry-pit model with one bulk phase can be transformed to an interphase model in a similar way as the Boolean model and the hard spheres one. However, expression (14) for the volume fraction of pores for the cherry-pit model does not depend explicitly on the pore size. Remembering that the hard core radius $r_{h}$ and the corresponding pore radius $r_{p}$ are related by

$$
r_{h}=\lambda r_{p}
$$

and considering two monodisperse cherry-pit models 1 and 2 characterized by the same values of $\phi_{h}$ and $r_{h}$ but different pore size $r_{p 1}, r_{p 2}$ with $r_{p 2}=r_{p 1}-\delta t$ and $\lambda_{1}=r_{h} / r_{p 1}, \lambda_{2}=$ $r_{h} / r_{p 2}$, the volume fraction of the interphase of the corresponding cherry-pit interphase models is

$$
c_{i}=V_{V}\left(\phi_{h}, \lambda_{1}\right)-V_{V}\left(\phi_{h}, \lambda_{2}\right), \quad \lambda_{2}=\frac{\lambda_{1}}{1-\delta t / r_{p 1}} .
$$

$$
\begin{aligned}
\text { Setting } \lambda_{1}= & \lambda, \delta t=r \delta \lambda / \lambda \text {, replacing } \\
& \frac{V_{V}\left(\phi_{h}, \lambda\right)-V_{V}\left(\phi_{h}, \lambda+\delta \lambda\right)}{\delta \lambda}
\end{aligned}
$$

by

$$
\frac{\partial V_{V}\left(\phi_{h}, \lambda\right)}{\partial \lambda}
$$

and carrying out $\delta \lambda \rightarrow 0$ one obtains from ((23), (25))

$$
c_{i}=r \frac{\delta \lambda}{\lambda} S_{V}=\delta t S_{V}
$$

which corresponds with (27).

3.5. Reconstruction. It is often important to generate models not from mathematical algorithms or rules but from experimentally obtained structural information. Such information is basically incomplete for random systems. The generation of models from experimental data or reconstruction must therefore focus on reproducing important structure parameters like volume fraction, pore size distribution, or correlation functions. The problem of reconstruction is related to the well-known field of stereology (see chapter 10 in [10]). A typical problem of stereology is, for example, to estimate the size distribution of particles from the length distribution of chords inside particles where the chords are determined from linear sections of the considered sample. Few problems are exactly solved, for example, the determination of the size distribution of pores from planar sections of the sample if 
the pores have spherical shape. The size distribution of the pores determines, however, not yet the spatial distribution of the spheres. In this case, small-angle scattering data would be helpful to complete information about spatial correlations of the 3-dimensional arrangement of the pores.

Procedures for the reproduction of random structures have been successfully developed in recent years. Roberts generated 3-dimensional models from 2-dimensional images [49]. Yeong and Torquato [50, 51] proposed a procedure applicable to very different types of random media. Hilfer and Manwart [52] used physical properties like conductivity and permeability in order to improve reconstruction. Reconstruction from microtomographic images was carried out by Øren and Bakke [53]. Ohser and Schladitz [14] presented general methods of computational image analysis. Methods for the reconstruction of random media of the type described by the Poisson grain model have been developed by Arns et al. $[54,55]$. Problems occurring with reconstruction on the nanometer scale have been addressed by Prill et al. [56].

\section{Structure-Property Relationships}

Properties of random heterogeneous materials including porous media have been considered in many theoretical studies. The effective medium theory see, for example, $[4,5]$, and its combination with the maximum entropy approach [57] have been proven as a powerful tool to understand macroscopic properties, and various structure models have been considered for the study of structure-property relations of heterogeneous materials [6, 7, 58-60]. Among the different structure models, the Poisson grain model appears as one of the most successful approaches for establishing structureproperty relationships of random media [58, 61-66]. Recent work on the optimization of property combinations of random media by means of structure variation can be found in $[67,68]$.

We consider the composite sphere assemblage model (see, e.g., [4]) and related effective medium theories (see, e.g., [5]) as most adequate for the models described in the previous sections. Explicit expressions for dielectric constants, thermal conductivity, and isotropic elastic moduli of models consisting of a matrix and spherical inclusions have been derived and discussed in $[4,5,67-69]$. Below, the idea of this approach is explained for the example of the thermal conductivity of a porous medium with coated pore surface.

A corresponding composite sphere element (CSE) is made up by a central sphere with radius $r_{p}$ filled with phase $p$, a concentric spherical shell of phase $i$ with thickness $r_{i}-r_{p}$, and a second shell of phase $b$ with thickness $r_{b}-r_{i}$ (see Figure 9). In our case, phase $p$ represents the pore content (vacuum, air, or something else), phase $i$ is the pore coating layer, and phase $b$ is the bulk material of the porous medium. The CSE is embedded in the effective medium. The volume fraction of the pores, the coating, and the bulk material are denoted by $c_{p}, c_{i}$, and $c_{b}$, respectively. Writing

$$
r_{p i}=\left(\frac{r_{p}}{r_{i}}\right)^{3}, \quad r_{i b}=\left(\frac{r_{i}}{r_{b}}\right)^{3}, \quad r_{p b}=\left(\frac{r_{p}}{r_{b}}\right)^{3}
$$

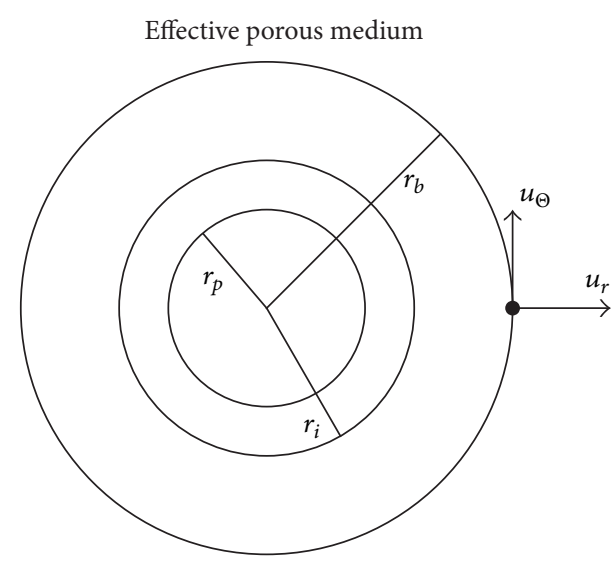

FIgURE 9: Composite sphere element.

the volume fractions of the phases are

$$
c_{p}=r_{p b}, \quad c_{i}=r_{i b}-r_{p b}, \quad c_{b}=1-r_{i b} .
$$

The temperature distribution in the porous medium follows the Laplace equation

$$
\Delta T=0
$$

And the corresponding intensity field is

$$
\mathbf{f}=-\nabla T
$$

Heat flux $\mathbf{j}$ and thermal conductivity $\sigma$ are defined by

$$
\mathbf{j}=\sigma \mathbf{f} \text {. }
$$

According to [70] the solution of the Laplace (43) for the CSE unit embedded in the effective medium is

$$
\mathbf{f}= \begin{cases}C_{p} \sin \Theta \mathbf{u}_{\Theta}-C_{p} \cos \Theta \mathbf{u}_{r}, & 0 \leq r \leq r_{p}, \\ \left(C_{i}+\frac{D_{i}}{r^{3}}\right) \sin \Theta \mathbf{u}_{\Theta} & \\ +\left(-C_{i}+2 \frac{D_{i}}{r^{3}}\right) \cos \Theta \mathbf{u}_{r}, & r_{p} \leq r \leq r_{i}, \\ \left(C_{b}+\frac{D_{b}}{r^{3}}\right) \sin \Theta \mathbf{u}_{\Theta} & \\ +\left(-C_{b}+2 \frac{D_{b}}{r^{3}}\right) \cos \Theta \mathbf{u}_{r}, & r_{i} \leq r \leq r_{b}, \\ -f_{0} \sin \Theta \mathbf{u}_{\Theta}+f_{0} \cos \Theta \mathbf{u}_{r}, & r_{b} \leq r .\end{cases}
$$

The boundary conditions of the heat flux through the interfaces pore to coating, coating to bulk, and bulk to effective medium require continuity of the radial component $\mathbf{j u}_{r}$ of the heat flux, $\mathbf{j}$, and of the tangential component $\mathbf{f} \mathbf{u}_{\Theta}$ of the temperature gradient, $-\mathbf{f}$. Denoting the values of the thermal conductivity of the pore phase, the coating interlayer, the bulk 
TABLE 1: Mathematically equivalent properties (according to [5]).

\begin{tabular}{lcccc}
\hline Problem & Potential & Field & Flux & Property \\
\hline $\begin{array}{l}\text { Heat conduction } \\
\text { Electrical }\end{array}$ & Temperature $T$ & Temperature gradient $-\nabla T$ & Heat flux f & Heat conductivity $\sigma$ \\
conductivity & Electrical potential & Electric field & Current density & Electrical \\
Diffusion & Density & Density gradient & Current density & Diffusion constant \\
Electrostatics & Electric potential & Electric field & Electric displacement & Dielectric constant \\
Magnetostatics & Magnetic potential & Magnetic field & Magnetic induction & Permeability \\
\hline
\end{tabular}

material, and the effective medium by $\sigma_{p}, \sigma_{i}, \sigma_{b}$, and $\sigma^{*}$ one obtains a system of linear equations

$$
\left(\begin{array}{cccccc}
1 & -r_{p i} & -1 & 0 & 0 & 0 \\
-\sigma_{p} & r_{p i} \sigma_{i} & -2 \sigma_{i} & 0 & 0 & 0 \\
0 & 1 & 1 & -r_{i b} & -1 & 0 \\
0 & -\sigma_{i} & 2 \sigma_{i} & r_{i b} \sigma_{b} & -2 \sigma_{b} & 0 \\
0 & 0 & 0 & 1 & 1 & 1 \\
0 & 0 & 0 & -\sigma_{b} & 2 \sigma_{b} & -\sigma^{*}
\end{array}\right)\left(\begin{array}{c}
C_{p} \\
C_{i} \\
D_{i} \\
C_{b} \\
D_{b} \\
f_{0}
\end{array}\right)=0
$$

This system has a solution if the determinant of the coefficient matrix vanishes. Solving the resulting equation for $\sigma^{*}$ one obtains for the effective thermal conductivity

$$
\sigma^{*}=\sigma_{b}+\frac{r_{i b}}{\left(1-r_{i b}\right) / 3 \sigma_{b}-1 /\left(\sigma_{b}-\sigma^{\#}\right)}
$$

with

$$
\sigma^{\#}=\sigma_{i}+\frac{r_{p i}}{\left(1-r_{p i}\right) / 3 \sigma_{i}-1 /\left(\sigma_{i}-\sigma_{p}\right)} .
$$

Quantity $\sigma^{\#}$ can be interpreted as the effective conductivity of a subsystem consisting of spherical pores with conductivity $\sigma_{p}$ embedded in a matrix with property $\sigma_{i}$ and the corresponding volume fractions.

It is known that a number of physical problems are mathematically equivalent to the formulation of thermal conductivity [5]. Therefore, (43) to (49) apply also to electrical conductivity, static dielectric property, magnetostatics, and diffusion (see Table 1).

The elastic moduli of the considered model can be calculated using either the CSA model [4] or the extended replacement method [71]. The results can be expressed in a similar form as expressions ((48), (49)). Denoting the effective property of the system by $a^{*}$, one can write $[48,68$, 69]:

$$
\begin{gathered}
\frac{a^{*}}{a_{b}}=1+\frac{1-c_{b}}{\alpha_{b}^{a} c_{b}-a_{b} /\left(a_{b}-a^{\#}\right)}, \\
\frac{a^{\#}}{a_{i}}=1+\frac{1-c^{\#}}{\alpha_{i}^{a} c^{\#}-a_{i} /\left(a_{i}-a_{p}\right)}, \\
c^{\#}=\frac{c_{p}}{c_{p}+c_{i}},
\end{gathered}
$$

$$
\alpha_{m}^{a}= \begin{cases}\frac{1}{3}, & a=\sigma \\ \frac{3 K_{m}}{3 K_{m}+4 G_{m}}, & a=K \\ \frac{6\left(K_{m}+2 G_{m}\right)}{5\left(3 K_{m}+4 G_{m}\right)}, & a=G .\end{cases}
$$

The symbol $\sigma$ stands for dielectric constant, thermal conductivity, and related properties, $K, G$ denote elastic bulk and shear modulus for isotropic systems, and index $m=p, i, b$ stands for pore, interphase layer, and bulk phase.

Expressions ((50) to (53)) are rigorous results obtained for geometrical situations as given in the structure models described above. The results for the properties are unique and no uncertainty due to unknown topology of phase distribution occurs. Nevertheless there are relations to the Hashin-Sthrikman bounds for properties of heterogeneous materials [72] which we discuss here for the case of the dielectric constant $k$. For simplicity, we use a model consisting of a matrix and spherical inclusions. Choosing $c_{i}=0$ with $c_{p}=1-c_{b}, a=k$, and $\alpha^{k}=1 / 3$, assuming $k_{b}<k_{p}$, and changing the topology of the system by interchanging properties and volume fractions of matrix and inclusions, that is, $k_{b} \leftrightarrow k_{p}, c_{b} \leftrightarrow c_{p}$, one obtains from ((50), (51)) the HashinSthrikman bounds for the effective dielectric constant, $k^{*}$, of a two-component composite (see also [5], (2.3))

$$
\begin{gathered}
k_{b}+\frac{k_{b} c_{p}}{c_{p} / 3-k_{p} /\left(k_{p}-k_{b}\right)} \leq k^{*} \leq k_{p} \\
+\frac{k_{p} c_{b}}{c_{b} / 3-k_{b} /\left(k_{b}-k_{p}\right)} .
\end{gathered}
$$

The same procedure can be carried out for the HashinSthrikman bounds for the isotropic elastic moduli (see [4], 
Sections 3.2 and 4.1). For a given distribution of phases on pores, interphase layer, and bulk there is no uncertainty of the topology and, consequently, expressions ((50) to (53)) for the properties are unique.

\section{Applications}

5.1. Fractal-Like Pore Structure. Porous media may have fractal features (see, e.g., [73-75]). We consider here a model for surface fractals which is based on the Boolean model described in Section 3.1. This model is tunable with respect to the fractal dimension of the internal surface, and explicit expression for the geometrical characteristics defined in Section 2 is known $[68,76]$. The size distribution of spheres used for the construction of the model is defined by selfsimilarity rules for the radii $r_{u}$ and the partial number density $v_{u}$ of spheres $r_{u}$ :

$$
r_{u}=u^{s} r_{1}, \quad v_{u}=u^{-t} v_{1}, \quad u_{0} \leq u \leq 1,
$$

where $r_{u}$ and $v_{u}$ are radius and number density of spheres belonging to the interval $(u-d u, u)$. Parameter $\kappa=r_{0} / r_{1}=u_{0}^{s}$ describes the ratio of minimum and maximum sphere size. The normalized size distribution $f(r)$ is then given by

$$
\begin{gathered}
f(r)= \begin{cases}\frac{\eta \kappa^{\eta}}{\left(1-\kappa^{\eta}\right) r_{1}}\left(\frac{r}{r_{1}}\right)^{-\eta-1}, & r_{0} \leq r \leq r_{1} \\
0, & \text { otherwise }\end{cases} \\
\eta=\frac{t-1}{s} .
\end{gathered}
$$

Number density of spheres, volume fraction, specific surface area, and correlation function are given by ((14), (15), and (16)) and

$$
\begin{gathered}
\nu=\nu_{1} \frac{1-\kappa^{\eta}}{\kappa^{\eta} \eta s}, \\
\nu \bar{V}=\nu_{1} \frac{4 \pi}{3} r_{1}^{3} \frac{1-\kappa^{3-\eta}}{(3-\eta) s}, \\
\nu \bar{S}=\nu_{1} 4 \pi r_{1}^{2} \frac{\kappa^{2-\eta}-1}{(\eta-2) s}, \\
\times\left\{\frac{1-\left(\rho / r_{1}\right)^{3-\eta}}{3-\eta}+\frac{3}{2}\left(\frac{r}{2 r_{1}}\right) \frac{1-\left(\rho / r_{1}\right)^{2-\eta}}{\eta-2}\right. \\
\frac{1-\kappa^{3-\eta}}{\ln \frac{1}{1-V_{V}}} \\
\left.\left.\frac{r}{2 r_{1}}\right)^{3} \frac{1-\left(\rho / r_{1}\right)^{-\eta}}{\eta}\right\}, \\
0 \leq r \leq 2 r_{1},
\end{gathered}
$$

where

$$
\rho= \begin{cases}r_{0}, & \frac{r}{2}<r_{0} \\ \frac{r}{2}, & r_{0} \leq \frac{r}{2} \leq r_{1}\end{cases}
$$

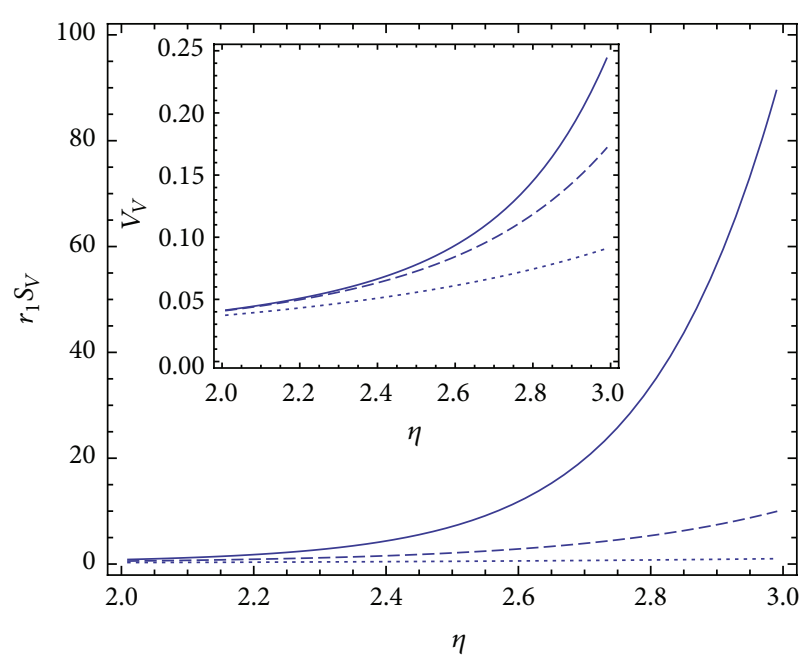

FIGURE 10: Volume fraction (insert) and specific surface area of selfsimilar Boolean models versus self-similar parameter $\eta ; r_{1}=1, v_{1}=$ 0.01 , and $s=1 ; \kappa=0.1$ (dotted), 0.01 (dashed), and 0.001 (solid line).

The self-similar model described by ((14), (15), and (16)) and ((58), (59), (and 60)) exhibits the properties of a surface fractal if the specific surface area tends to infinity while the volume fraction remains finite for $\kappa \rightarrow 0$. This is the case for

$$
\begin{gathered}
2 \leq \eta<3, \\
d_{f}=\lim _{\epsilon \rightarrow 0} \frac{\ln \left(P\left(\mathbf{r} \in S_{\epsilon}\right) / \epsilon^{3}\right)}{\ln (1 / \epsilon)}=\eta
\end{gathered}
$$

is the dimension of the fractal internal surface of the model according to the Minkowski-Bouligand definition [77, 78]. Quantity $S_{\epsilon}$ is the same as in (8). Fractality of a porous medium can be detected by means of small-angle scattering methods [73]. In such cases the limit $r / r_{1} \ll 1$ is of interest which determines the behavior of the scattering intensity at large scattering angles. One obtains from ((7), (60))

$$
C(r)-V_{V}^{2} \propto\left(\frac{r}{2 r_{1}}\right)^{3-d_{f}}
$$

which is in accordance with [73, 79].

The behavior of the self-similar system is illustrated by Figures 10 and 11. The volume fraction of pores increases with increasing values of the self-similarity parameter $\eta$ and also with decreasing ratio of radii $\kappa$ of smallest to largest pores (Figure 10). Of course, $V_{V}$ keeps limited in the fractal limit $\kappa \longrightarrow 0$, where $\eta$ takes the meaning of the fractal dimension of the internal surface (see expressions ((14), (58)). In the extreme case of $\kappa=0$ and $d_{f} \longrightarrow 3$ the porosity $V_{V}$ tends to 1 . The specific surface area given by ((15), (59)) is controlled by the term $\kappa^{2-\eta}$. Clearly, $S_{V}$ tends to infinity for $\kappa \longrightarrow 0$ independent of the value $2<d_{f}<3$ of the fractal dimension of the internal surface. The behavior of $S_{V}$ in the fractal limit appears also applying (9) to (63). Obviously, $S_{V}$ tends to infinity for each fractal dimension $2<d_{f}<3$ of the 


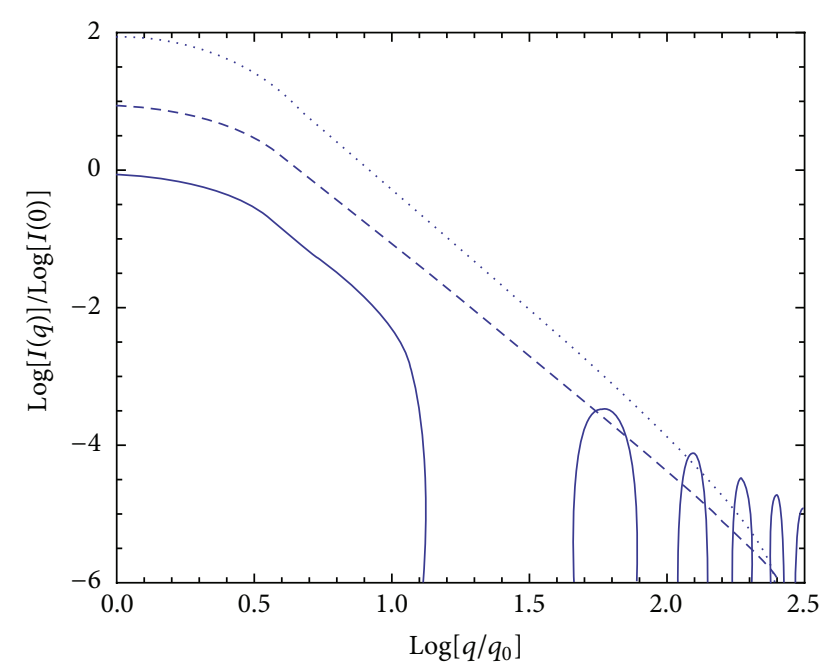

FIGURE 11: Small-angle scattering intensity of self-similar Boolean models; $q_{0}=1, r_{1}=1, v_{1}=0.05, s=1 ; \kappa=0.001$, and $\eta=2.1$ (dotted, curve shifted on the ordinate by 2$) ; \eta=2.1$ (dashed, shifted by 1$) ; \kappa=0.05$, and $\eta=2.5$ (solid line).

internal surface. Figure 10 illustrates the dependence of $S_{V}$ on the self-similarity parameter $\eta$ for different values of $\kappa$.

The scattering intensity of the self-similar system can be calculated $[11,68,78]$ using

$$
I(q)=4 \pi \int_{0}^{\infty} r^{2}\left[C(r)-V_{V}^{2}\right] \frac{\sin (q r)}{q r} d r
$$

where $q$ is the absolute value of the scattering vector defined in the usual way [74]. If the size of pores is on the nanometer scale, $I(q)$ is called small-angle scattering intensity. Inserting ((16), (60)) in (64) an explicit expression for the small-angle scattering intensity can be obtained. The limit of small $r$ values given in (63) becomes apparent in $I(q)$ in the form

$$
I(q) \propto q^{6-d_{f}}
$$

in the fractal limit for not to small $q$-values.

Figure 11 shows scattering intensities of a self-similar system with fractal-like behavior for small $\kappa$. The scattering intensity is dominated in the region $\log \left(q / q_{0}\right)<0.5$ by the largest pores of the system $\left(r_{1}=1\right.$ in suitable units, e.g., in nanometers). The curve for the system with small range $\kappa=0.05$ of self-similarity follows approximately expression (65) in the interval $0.5<\log \left(q / q_{0}\right)<1$, and for $\log (q)>1$ the scattering intensity $I(q)$ is determined by the smallest pores of size $r_{0}=\kappa r_{1}$. Systems showing such scattering curves are sometimes called fractal-like. Seriously speaking, the system is self-similar over about one order of magnitude of length scale. The curves for $\kappa=0.001$ represent systems which are self-similar over 3 orders of magnitude. This is reflected by the linear behavior in the $\log (I(q))$ versus $\log \left(q / q_{0}\right)$ plot for $0.5<\log \left(q / q_{0}\right)<2.3$ where the limit $(65)$ applies. Here, the term fractal-like would be justified.
5.2. Optimization of Porous Dielectric Materials. The improvement of silicon based microelectronic devices like microprocessors has been a continuing process over decades [80]. This includes the further development of many components and also the replacement of components by new materials, for example, the replacement of aluminum wires for connecting billions of transistors in a microprocessor circuit by copper wires. This replacement was necessary because the performance of microprocessors is increasingly affected by the on-chip interconnect design and technology. Signal delay caused by the resistor-capacitor (RC) interaction in the system of copper wires and insulating layers becomes more and more important compared to the transistors' signal delay [81, 82]. Additionally, cross-talking and power dissipation worsen due to increasing RC coupling caused by continuous reduction of device dimensions [83, 84]. The introduction of the copper technology was one step to reduce these unfavorable implications of continuous miniaturization. Another one concerns the reduction of the dielectric constant of the insulating layers separating the copper wires. Manufacturable materials with effective dielectric constants of 2.90 to 2.60 are available at the present time; however, feasible solutions with $k<2.60$ are difficult to obtain [85].

Research is in progress to define dielectric materials with extremely low dielectric constant. Besides dense low$k$ materials like organosilicate glass and the challenging airgap technology, insulating materials with porosity on the nanometer scale are considered as a promising way to reduce the above problems [85]. Among possible nanoporous insulating materials [86-88] nanoporous silica has the advantage that it can be implemented comparably easily in the current silicon technology.

We consider the following problem: optimize the structure of nanoporous silica in such a way that the dielectric constant $k$ is as low as possible, for example, less than 2 (vacuum: $k=1, \mathrm{SiO}_{2}: k=4$ ), and the Young's modulus $E$ as high as possible, for example, higher than $5 \mathrm{GPa}\left(\mathrm{SiO}_{2}\right.$ : $E=74 \mathrm{GPa}$ ) which would match future technological requirements. Using an interphase model specified by the volume fractions of pores, $c_{p}$, and interphase, $c_{i}$, and choosing a favorable second material additionally to silica, effective properties can be calculated for the models with ((50), (51), and (52)) and (53) with the corresponding values for $\alpha_{a}^{k}$; the Young's modulus is given by

$$
E=\frac{9 K G}{3 K+G}
$$

for isotropic systems. The properties of the components used for the simulations are quoted in Table 2. Figure 12 shows contour plots of effective property combinations in the plane spanned by $c_{i}$ and $c_{p}$ for a model consisting of porous Parylene coated with $\mathrm{SiO}_{2}$. Due to the porosity and the low $k$-value of the bulk material very low values of the dielectric constant can be achieved. However, the Young's modulus is too small for pure porous Parylene. Coating with $\mathrm{SiO}_{2}$ improves the effective modulus with the result that for $c_{i} \approx 0.2$ and pore fraction of at least 0.65 excellent values for $k^{*}<1.5$ can be combined with reasonable values $E^{*} \geq 5 \mathrm{GPa}$. The required 
TABLE 2: Properties of the structural components for models of coated porous ultralow $k$ dielectrics.

\begin{tabular}{lccc}
\hline Component & $K(\mathrm{GPa})$ & $G(\mathrm{Gpa})$ & $k$ \\
\hline Parylene & 4.7 & 1.0 & 2 \\
$\mathrm{SiO}_{2}$ & 36.7 & 31.2 & 4 \\
Vacuum & 0 & 0 & 1 \\
\hline
\end{tabular}

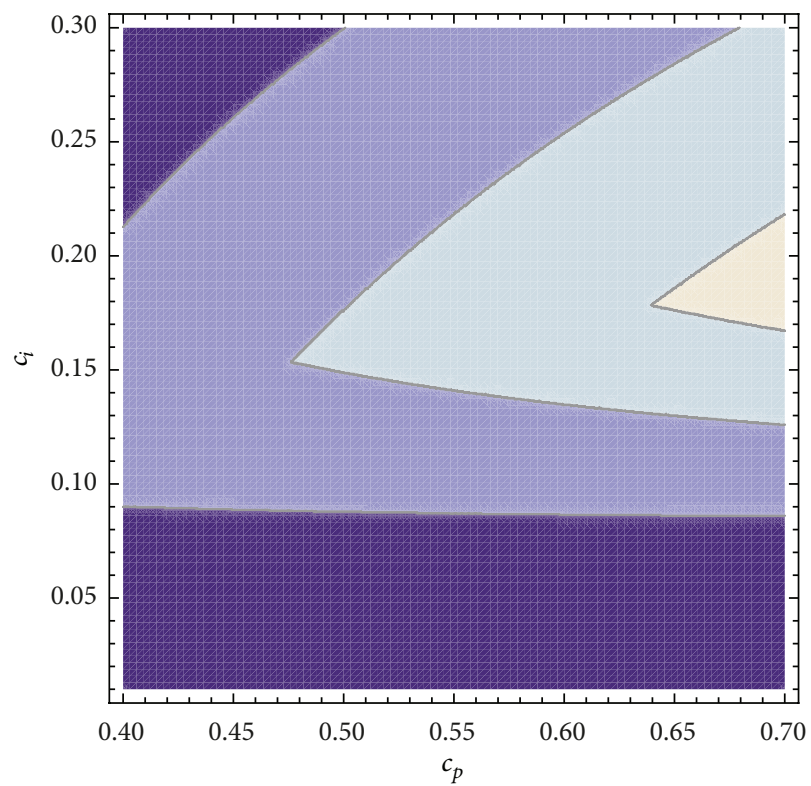

FIGURE 12: Effective property map for porous Parylene coated with $\mathrm{SiO}_{2}$ versus volume fractions $c_{p}$ and $c_{c}$ of pores and coating layer, respectively. Properties of the components are given in Table 2. Regions with $k^{*}<1.5, E^{*}>5 \mathrm{GPa}$ (yellow), $k^{*}<1.6, E^{*}>4 \mathrm{GPa}$ (light blue), $k^{*}<1.7, E^{*}>3 \mathrm{GPa}$ (blue), and $k^{*} \geq 1.7, E^{*} \leq 3 \mathrm{GPa}$ (dark blue).

porosity of at least 0.65 is, however, very high and cannot be achieved with random hard sphere packing models with equal spheres. Instead, the pores must have a polydisperse size distribution to obtain $c_{p}>0.65$. The parameters of the required size distribution can be determined using the methods described in Section 3; see also Figure 2.

Starting with porous silica and coating it with Parylene another effective property map appears (Figure 13). Systems with an effective dielectric constant of $k^{*}<1.8$ can be achieved at moderate porosity of about 0.60 for values of the effective Young's modulus of not less than $12 \mathrm{GPa}$, which appears as an excellent combination of dielectric and mechanical properties for the purpose under consideration.

5.3. Simulation of Coating Processes. The previous section showed for a specific case that introducing an interphase layer into a porous system may come out as a useful method to improve effective properties of a porous system. Coating of porous media has been proven to be a successful method to modify their properties in many fields of application. This applies not only to the properties discussed above but also

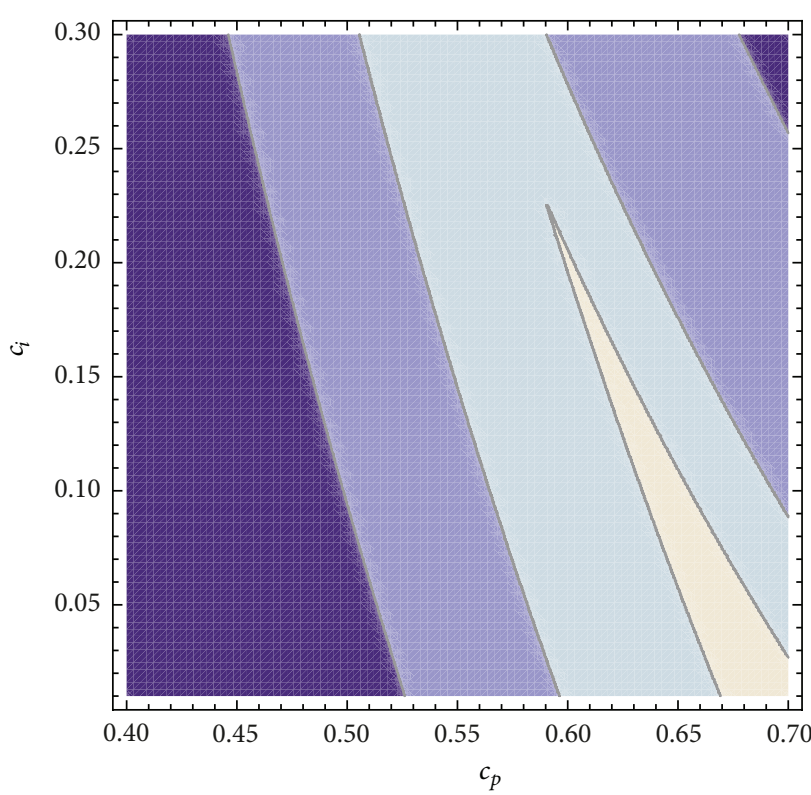

FIGURE 13: Effective property map for porous $\mathrm{SiO}_{2}$ coated with Parylene versus volume fractions $c_{p}$ and $c_{i}$ of pores and coating layer, respectively. Properties of the components are given in Table 2. Regions with $k^{*}<1.8, E^{*}>12 \mathrm{GPa}$ (yellow), $k^{*}<2.0, E^{*}>10 \mathrm{GPa}$ (light blue), $k^{*}<2.2, E^{*} \leq 5 \mathrm{GPa}$ (blue), and $k^{*} \geq 2.2, E^{*} \leq 5 \mathrm{PGa}$ (dark blue).

to flow and transport in porous media [7], percolation [89], enzyme immobilization $[90,91]$, adsorption processes [9294], and others. Different methods of coating and surface refinement of open-pore materials are known, for example, spray coating [95] and microarc oxidation coating [96] of metallic foams [97], and atomic layer deposition [15, 98-101]. The latter method is of special interest for media which are characterized by open-porosity on the nanometer scale.

We study the growth of a layer on the internal surface of open-pore systems within the framework of the cherry-pit model. Expression (37) for the volume fraction of a coating layer is written in the form

$$
c_{i}=V_{V}\left(\lambda_{0}\right)-V_{V}\left(\lambda_{\tau}\right)
$$

where $\tau$ denotes the coating time. For simplicity we consider a system with narrow pore size distribution. In a continuous coating process the radius $r_{\tau}$ of a given pore reduces according to

$$
r\left(\lambda_{\tau}\right)=r_{0}-\mu r_{h} \tau=\frac{r_{h}}{\lambda_{\tau}}, \quad r_{0}=\frac{r_{h}}{\lambda_{0}}
$$

One obtains

$$
\lambda_{\tau}=\frac{\lambda_{0}}{1-\lambda_{0} \mu \tau}
$$

where $\mu$ is the growth rate of layer thickness measured in units of the pit radius of the cherry-pit model. For discontinuous processes like atomic layer deposition, $\mu \tau$ has to be replaced by $\Delta t n$, where $n$ is the number of the deposition cycle and 


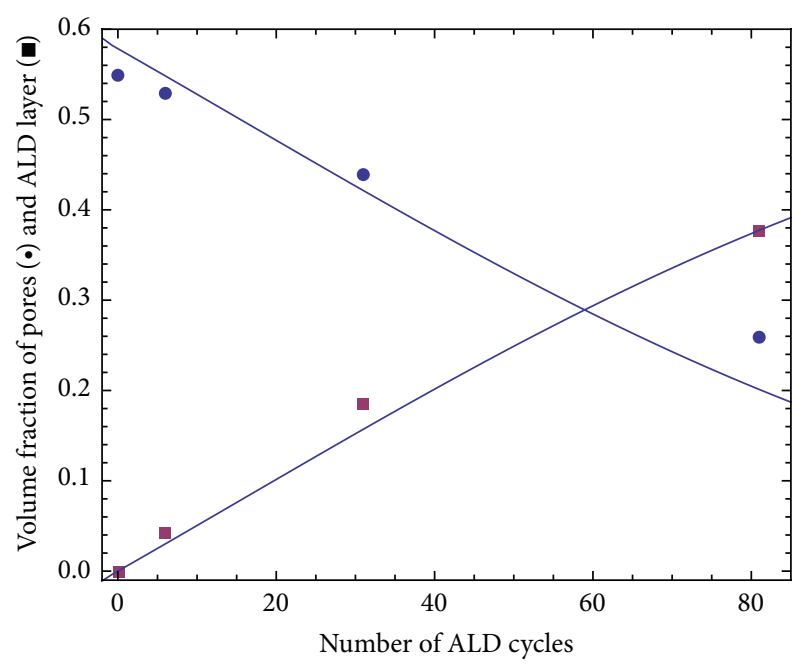

FIGURE 14: Volume fraction of pores (circles) and ALD layer (squares) of $\mathrm{Al}_{2} \mathrm{O}_{3}$ coated polypropylene. Experimental data points taken from [15]. Curves calculated using formula (36).

TABLE 3: Properties of the structural components used for the atomic layer deposition process.

\begin{tabular}{lccc}
\hline Component & $K(\mathrm{GPa})$ & $G(\mathrm{Gpa})$ & $\sigma(\mathrm{W} / \mathrm{mK})$ \\
\hline Polypropylene & 6 & 0.6 & 0.12 \\
$\mathrm{Al}_{2} \mathrm{O}_{3}$ & 165 & 124 & 18 \\
\hline
\end{tabular}

$\Delta t$ is the increment of layer thickness achieved by one cycle measured in units of the pit radius. We apply the present approach to experimental results for atomic layer deposition of $\mathrm{Al}_{2} \mathrm{O}_{3}$ on porous polypropylene [15]. Figure 14 compares the evolution of the porosity and the volume fraction of the $\mathrm{Al}_{2} \mathrm{O}_{3}$ layer with the number of deposition cycles. The cycle numbers are corrected for the induction period [15]. The model curves reproduce the trend of the experimental data with an accuracy of about $20 \%$.

The estimated volume fractions can now be used to predict effective properties of the coated material. Using known data (see Table 3 ) for bulk, $K$, shear, $G$, modulus, and the thermal conductivity $\sigma$ of polypropylene and for $\mathrm{Al}_{2} \mathrm{O}_{3}$ the effective elastic and thermophysical properties of the coated material are calculated. Figure 15 shows the results for the effective thermal conductivity and the Young's modulus. Both for the Young's modulus and the thermal conductivity the essential increase is achieved within the first 20 deposition cycles. These results supplement the experimental data obtained in [15] by predicting the change of physical properties during the deposition process.

\section{Conclusion}

There are appropriate theoretical tools for a consistent description of the structure and effective properties of isotropic random porous media. These tools provide impr-oved facilities to develop suitable models for porous materials from experimentally obtained structural parameters and to optimize

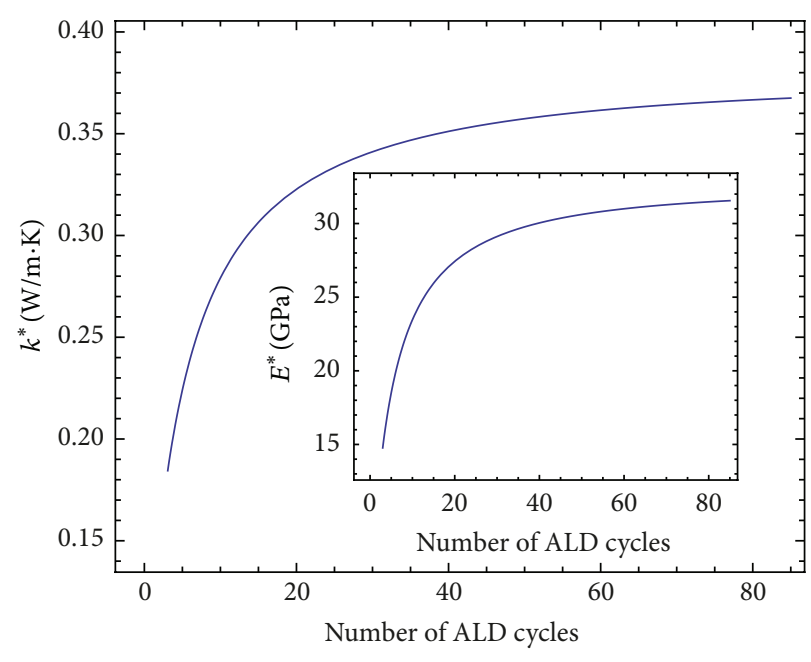

FIGURE 15: Effective thermal conductivity and Young's modulus of $\mathrm{Al}_{2} \mathrm{O}_{3}$ coated polypropylene versus ALD cycle number.

effective properties by simulating the response of effective properties on structural modification.

\section{Conflict of Interests}

The authors declare that there is no conflict of interests regarding the publication of this paper.

\section{References}

[1] J. M. Ziman, Models of Disorder, Cambridge University Press, Cambridge, UK, 1979.

[2] N. E. Cusack, The Physics of Structurally Disordered Matter, Adam Hilger, Bristol, UK, 1987.

[3] D. Weaire, The Physics of Foam, Clarendon Press, Oxford, UK, 1999.

[4] W. Kreher and W. Pompe, Internal Stresses in Heterogeneous Solids, Akademie-Verlag, Berlin, Germany, 1999.

[5] T. C. Choy, Effective Medium Theory-Principles and Applications, Clarendon Press, Oxford, UK, 1999.

[6] S. Torquato, Random Heterogeneous Materials: Microstructure and Macroscopic Properties, Springer, New York, NY, USA, 2002.

[7] M. Sahimi, Flow and Transport in Porous Media and Fractured Rock: From Classical Methods to Modern Approaches, WileyVCH, Weinheim, Germany, 1995.

[8] D. Stoyan, W. S. Kendall, and J. Mecke, Stochastic Geometry and Its Applications, Akademie-Verlag, Berlin, Germany, 1987.

[9] D. Stoyan, W. S. Kendall, and J. Mecke, Stochastic Geometry and Its Applications, Wiley, Chichester, UK, 2nd edition, 1995.

[10] S. N. Chiu, D. Stoyan, W. S. Kendall, and J. Mecke, Stochastic Geometry and Its Applications, Wiley, Weinheim, Germany, 3rd edition, 2013.

[11] H. Hermann, Stochastic Models of Heterogeneous Materials, Trans Tech, Zurich, Switzerland, 1991.

[12] K. R. Mecke and D. Stoyan, Eds., Statistical Physics and Spatial Statistics-The Art of Analyzing and Modeling Spatial Structures and Pattern Formation, Springer, Berlin, Germany, 2000. 
[13] J. Ohser and F. Mücklich, 3D Images of Materials Structures: Processing and Analysis, Wiley- $\mathrm{VCH}$, Weinheim, Germany, 2000.

[14] J. Ohser and K. Schladitz, 3D Images of Materials and Structures: Processing and Analysis, Wiley-VCH, Weinheim, Germany, 2009.

[15] S. Y. Jung, A. S. Cavanagh, L. Gevilas et al., "Improved functionality of lithium-ion batteries enabled by atomic layer deposition on the porous microstructure of polymer separators and coating electrodes," Advanced Energy Materials, vol. 2, no. 8, pp. 1022-1027, 2012.

[16] R. Hosemann and S. N. Bagchi, Direct Analysis of Diffraction by Matter, North-Holland, Amsterdam, The Netherlands, 1962.

[17] G. Porod, "Die Röntgenkleinwinkelstreuung von dichtgepackten kolloiden Systemen," Kolloid-Zeitschrift, vol. 125, no. 1, pp. 51-57, 1952.

[18] C. Kittel, Introduction to Solid State Physics, John Wiley \& Sons, New York, NY, USA, 1996.

[19] J. D. Bernal and J. Mason, "Packing of spheres: co-ordination of randomly packed spheres," Nature, vol. 188, no. 4754, pp. 910911, 1960.

[20] G. S. Cargill III, "Structure of metallic alloy glasses," Solid State Physics, vol. 30, pp. 227-320, 1975.

[21] J. L. Finney, "Modelling the structures of amorphous metals and alloys," Nature, vol. 266, no. 5600, pp. 309-314, 1977.

[22] M. R. Hoare, "Packing models and structural specificity," Journal of Non-Crystalline Solids, vol. 31, no. 1-2, pp. 157-179, 1978.

[23] H. L. Löwen, "Fun with hard spheres," in Statistical Physics and Spatial Statistics-The Art of Analyzing and Modeling Spatial Structures and Pattern Formation, K. R. Mecke and D. Stoyan, Eds., pp. 295-331, Springer, Berlin, Germany, 2000.

[24] W. S. Jodrey and E. M. Tory, "Computer simulation of close random packing of equal spheres," Physical Review A, vol. 32, no. 4, pp. 2347-2351, 1985.

[25] J. Moscinski and M. Bargiel, "C-language program for the irregular close packing of hard spheres," Computer Physics Communications, vol. 64, no. 1, pp. 183-192, 1991.

[26] A. Bezrukov, M. Bargiel, and D. Stoyan, "Statistical analysis of simulated random packings of spheres," Particle \& Particle Systems Characterization, vol. 19, no. 2, pp. 111-118, 2002.

[27] A. Elsner, Computer-aided simulation and analysis of random dense packings of spheres [thesis], 2009 (German).

[28] B. Widom and J. S. Rowlinson, "New model for the study of liquid-vapor phase transitions," The Journal of Chemical Physics, vol. 52, no. 4, pp. 1670-1684, 1970.

[29] B. Widom, "Geometrical aspects of the penetrable-sphere model," The Journal of Chemical Physics, vol. 54, no. 9, pp. 39503957, 1971.

[30] C. N. Likos, K. R. Mecke, and H. Wagner, "Statistical morphology of random interfaces in microemulsions," The Journal of Chemical Physics, vol. 102, no. 23, pp. 9350-9361, 1995.

[31] K. R. Mecke, "A morphological model for complex fluids," Journal of Physics Condensed Matter, vol. 8, no. 47, pp. 96639667, 1996.

[32] F. H. Stillinger, "Phase transitions in the Gaussian core system," The Journal of Chemical Physics, vol. 65, no. 10, pp. 3968-3974, 1976.

[33] C. N. Likos, M. Watzlawek, and H. Löwen, "Freezing and clustering transitions for penetrable spheres," Physical Review E, vol. 58, no. 3, pp. 3135-3144, 1998.
[34] C. N. Likos, A. Lang, M. Watzlawek, and H. Löwen, “Criterion for determining clustering versus reentrant melting behaviour for bounded interaction potentials," Physical Review E, vol. 63, no. 3, Article ID 031206, 2001.

[35] C. N. Likos, B. M. Mladek, D. Gottwald, and G. Kahl, "Why do ultrasoft repulsive particles cluster and crystallize? Analytical results from density-functional theory," The Journal of Chemical Physics, vol. 126, no. 22, Article ID 224502, 2007.

[36] M.-J. Fernaud, E. Lomba, and L. L. Lee, "A self-consistent integral equation study of the structure and thermodynamics of the penetrable sphere fluid," The Journal of Chemical Physics, vol. 112, no. 2, pp. 810-816, 2000.

[37] A. Santos and A. Majewsky, "Fluids of spherical molecules with dipolarlike nonuniform adhesion: an analytically solvable anisotropic model," Physical Review E, vol. 78, no. 2, Article ID 021201, 2008.

[38] L. Blum and G. Stell, "Polydisperse systems-I. Scattering function for polydisperse fluids of hard or permeable spheres," The Journal of Chemical Physics, vol. 71, no. 1, pp. 42-46, 1979.

[39] P. A. Rikvold and G. Stell, "Porosity and specific surface for interpenetrable-sphere models of two-phase random media," The Journal of Chemical Physics, vol. 82, no. 2, pp. 1014-1020, 1985.

[40] P. A. Rikvold and G. Stell, " $D$-dimensional interpenetrablesphere models of random two-phase media: microstructure and an application to chromatography," Journal of Colloid and Interface Science, vol. 108, no. 1, pp. 158-173, 1985.

[41] S. Torquato, "Bulk properties of two-phase disordered mediaI. Cluster expansion for the effective dielectric constant of dispersions of penetrable spheres," The Journal of Chemical Physics, vol. 81, no. 11, pp. 5079-5088, 1984.

[42] S. Torquato, "Bulk properties of two-phase disordered mediaII. Effective conductivity of a dilute dispersion of penetrable spheres," The Journal of Chemical Physics, vol. 83, no. 9, pp. 4776-4785, 1985.

[43] S. Torquato, "Bulk properties of two-phase disordered mediaIII. New bounds on the effective conductivity of dispersions of penetrable spheres," The Journal of Chemical Physics, vol. 84, no. 11, pp. 6345-6359, 1986.

[44] K. Gotoh, M. Nakagawa, M. Furuuchi, and A. Yoshigi, "Pore size distributions in random assemblies of equal spheres," The Journal of Chemical Physics, vol. 85, no. 5, pp. 3078-3080, 1986.

[45] S. B. Lee and S. Torquato, "Porosity for the penetrableconcentric-shell model of two-phase disordered media: computer simulation results," The Journal of Chemical Physics, vol. 89, no. 5, pp. 3258-3263, 1988.

[46] D. Stoyan, A. Wagner, H. Hermann, and A. Elsner, "Statistical characterization of the pore space of random systems of hard spheres," Journal of Non-Crystalline Solids, vol. 357, no. 6, pp. 1508-1515, 2011.

[47] A. Elsner, A. Wagner, T. Aste, H. Hermann, and D. Stoyan, "Specific surface area and volume fraction of the cherry-pit model with packed pits," The Journal of Physical Chemistry B, vol. 113, no. 22, pp. 7780-7784, 2009.

[48] H. Hermann, A. Elsner, and D. Stoyan, "Surface area and volume fraction of random open-pore systems," Modelling and Simulation in Materials Science and Engineering, vol. 21, no. 8, Article ID 085005, 2013.

[49] A. P. Roberts, "Statistical reconstruction of three-dimensional porous media from two-dimensional images," Physical Review E, vol. 56, no. 3, pp. 3203-3212, 1997. 
[50] C. L. Y. Yeong and S. Torquato, "Reconstructing random media," Physical Review E, vol. 57, no. 1, pp. 495-506, 1998.

[51] C. L. Y. Yeong and S. Torquato, "Reconstructing random media-II. Three-dimensional media from two-dimensional cuts," Physical Review E, vol. 58, no. 1, pp. 224-233, 1998.

[52] R. Hilfer and C. Manwart, "Permeability and conductivity for reconstruction models of porous media," Physical Review E, vol. 64, no. 2, Article ID 021304, 4 pages, 2001.

[53] P.-E. Øren and S. Bakke, "Process based reconstruction of sandstones and prediction of transport properties," Transport in Porous Media, vol. 46, no. 2-3, pp. 311-343, 2002.

[54] C. H. Arns, M. A. Knackstedt, and K. R. Mecke, "Reconstructing complex materials via effective grain shapes," Physical Review Letters, vol. 91, no. 21, Article ID 215506, 4 pages, 2003.

[55] C. H. Arns, M. A. Knackstedt, and K. R. Mecke, "Boolean reconstructions of complex materials: integral geometric approach," Physical Review E, vol. 80, no. 5, Article ID 051303, 17 pages, 2009.

[56] T. Prill, K. Schladitz, D. Jeulin, M. Faessel, and C. Wieser, "Morphological segmentation of FIB-SEM data of highly porous media," Journal of Microscopy, vol. 250, no. 2, pp. 77-87, 2013.

[57] W. Kreher and W. Pompe, "Field fluctuations in a heterogeneous elastic material-an information theory approach," Journal of the Mechanics and Physics of Solids, vol. 33, no. 5, pp. 419-445, 1985.

[58] D. Jeulin, Morphologie mathématique et propriétés physiques des agglomérés de miinerai de fer et de coke métullirrgique [Ph.D. thesis], School of Mines, Paris, France, 1979.

[59] C. H. Arns, M. A. Knackstedt, W. V. Pinczewski, and K. R. Mecke, "Euler-Poincaré characteristics of classes of disordered media," Physical Review E, vol. 63, no. 3, Article ID 031112, 13 pages, 2001.

[60] C. H. Arns, M. A. Knackstedt, and K. R. Mecke, "Characterisation of irregular spatial structures by parallel sets and integral geometric measures," Colloids and Surfaces A, vol. 241, no. 1-3, pp. 351-372, 2004.

[61] H. Hermann and W. Kreher, "Structure, elastic moduli and internal stresses of iron-boron metallic glasses," Journal of Physics F: Metal Physics, vol. 18, no. 4, pp. 641-655, 1988.

[62] M. D. Rintoul and S. Torquato, "Precise determination of the critical threshold and exponents in a three-dimensional continuum percolation model," Journal of Physics A: Mathematical and General, vol. 30, no. 16, pp. L585-L592, 1997.

[63] A. P. Roberts and E. J. Garboczi, "Computation of the linear elastic properties of random porous materials with a wide variety of microstructure," Proceedings of the Royal Society A, vol. 458, no. 2021, pp. 1033-1054, 2002.

[64] K. Mecke and C. H. Arns, "Fluids in porous media: a morphometric approach," Journal of Physics Condensed Matter, vol. 17, no. 9, pp. S503-S534, 2005.

[65] F. Willot and D. Jeulin, hal-00553376, version 1-7, 2011.

[66] D. Jeulin, "Multi scale random sets: from morphology to effective behaviour," in Progress in Industrial Mathematics at ECMI 2010, M. Günther, A. Bartel, M. Brunk, S. Schöps, and M. Striebel, Eds., pp. 381-393, Springer, Berlin, Germany, 2012.

[67] S. Torquato, "Optimal design of heterogeneous materials," Annual Review of Materials Research, vol. 40, pp. 101-129, 2010.

[68] H. Hermann, "Effective dielectric and elastic properties of nanoporous low- $k$ media," Modelling and Simulation in Materials Science and Engineering, vol. 18, no. 5, Article ID 055007, 2010.
[69] V. Kokotin, H. Hermann, and J. Eckert, "Theoretical approach to local and effective properties of BMG based matrix-inclusion nanocomposites," Intermetallics, vol. 30, pp. 40-47, 2012.

[70] L. D. Landau and E. M. Lifschitz, Elektrodynamik der Kontinua, Akademie-Verlag, Berlin, Germany, 1967.

[71] I. Shen and J. Li, "Effective elastic moduli of composites reinforced by particle or fiber with an inhomogeneous interphase," International Journal of Solids and Structures, vol. 40, no. 6, pp. 1393-1409, 2003.

[72] Z. Hashin, "The elastic moduli of heterogeneous materials," Journal of Applied Mechanics, vol. 29, pp. 143-150, 1962.

[73] P. W. Schmidt, A. Hohr, H. B. Neumann, H. Kaiser, D. Avnir, and J. S. Lin, "Small-angle X-ray scattering study of the fractal morphology of porous silicas," The Journal of Chemical Physics, vol. 90, no. 9, pp. 5016-5023, 1989.

[74] P. W. Schmidt, "Small-angle scattering studies of disordered, porous and fractal systems," Journal of Applied Crystallography, vol. 24, pp. 414-435, 1991.

[75] B. Yu, "Analysis of flow in fractal porous media," Applied Mechanics Reviews, vol. 61, no. 5, Article ID 050801, 19 pages, 2008.

[76] H. Hermann and J. Ohser, "Determination of microstructural parameters of random spatial surface fractals by measuring chord length distributions," Journal of Microscopy, vol. 170, no. 1, pp. 87-93, 1993.

[77] U. Zähle, "Random fractals generated by random cutouts," Mathematische Nachrichten, vol. 116, no. 1, pp. 27-52, 1984.

[78] H. Hermann, "A new random surface fractal for applications in solid state physics," Physica Status Solidi B, vol. 163, no. 2, pp. 329-336, 1991.

[79] H. D. Bale and O. W. Schmidt, "Small-angle X-ray-scattering investigation of submicroscopic porosity with fractal properties," Physical Review Letters, vol. 53, no. 6, pp. 596-599, 1984.

[80] "International Technological Roadmap for Semiconductors," Interconnect. Semiconductor Industry Association, 2007, http//:www.itrs.net/.

[81] S. P. Jeng, R. H. Havemann, and M. C. Chang, "Process integration and manufacturasility issues for high performance multilevel interconnect," Materials Research Society Symposium Proceedings, vol. 337, article 25, 1994.

[82] S. H. Rhee, M. D. Radwin, J. I. Ng, and D. Erb, "Calculation of effective dielectric constants for advanced interconnect structures with low- $k$ dielectrics," Applied Physics Letters, vol. 83, no. 13, pp. 2644-2646, 2003.

[83] S. Yu, T. K. S. Wong, K. Pita, X. Hu, and V. Ligatchev, "Surface modified silica mesoporous films as a low dielectric constant intermetal dielectric," Journal of Applied Physics, vol. 92, no. 6, pp. 3338-3344, 2002.

[84] M. R. Wang, R. Rusli, M. B. Yu, N. Babu, C. Y. Li, and K. Rakesh, "Low dielectric constant films prepared by plasma-enhanced chemical vapor deposition from trimethylsilane," Thin Solid Films, vol. 462-463, pp. 219-222, 2004.

[85] K. Zagorodniy, D. Chumakov, C. Täschner et al., "Novel carboncage-based ultralow- $k$ materials: modeling and first experiments,' IEEE Transactions on Semiconductor Manufacturing, vol. 21, no. 4, pp. 646-660, 2008.

[86] J. L. Plawski, W. N. Gill, A. Jain, and S. Rogojevic, "Nanoporous dielectric films: fundamental property relations and microelectronics applications," in Interlayer Dielectrics for Semiconductor Technology, S. P. Murarka, M. Eizenberg, and A. K. Sinha, Eds., pp. 261-325, Elsevier, Amsterdam, The Netherlands, 2003. 
[87] K. Zagorodniy, H. Hermann, and M. Taut, "Structure and properties of computer-simulated fullerene-based ultralow- $k$ dielectric materials," Physical Review B, vol. 75, no. 24, Article ID 245430, 6 pages, 2007.

[88] K. Zagorodniy, G. Seifert, and H. Hermann, "Metal-organic frameworks as promising candidates for future ultralow- $k$ dielectrics," Applied Physics Letters, vol. 97, no. 25, Article ID 251905, 2010.

[89] J. Ohser, C. Ferrero, O. Wirjadi, A. Kuznetsova, J. Düll, and A. Rack, "Estimation of the probability of finite percolation in porous microstructures from tomographic images," International Journal of Materials Research, vol. 103, no. 2, pp. 184-191, 2012.

[90] H. Takahashi, B. Li, T. Sasaki, C. Miyazaki, T. Kajino, and S. Inagaki, "Catalytic activity in organic solvents and stability of immobilized enzymes depend on the pore size and surface characteristics of mesoporous silica," Chemistry of Materials, vol. 12, no. 11, pp. 3301-3305, 2000.

[91] J.-K. Kim, J.-K. Park, and H.-K. Kim, "Synthesis and characterization of nanoporous silica support for enzyme immobilization," Colloids and Surfaces A: Physicochemical and Engineering Aspects, vol. 241, no. 1-3, pp. 113-117, 2004.

[92] C. Vega, R. D. Kaminsky, and P. A. Monson, "Adsorption of fluids in disordered porous media from integral equation theory," The Journal of Chemical Physics, vol. 99, no. 4, pp. 30033013, 1993.

[93] G. V. Kapustin and J. Ma, "Modeling adsorption-desorption processes in porous media," Computing in Science \& Engineering, vol. 1, no. 1, pp. 84-91, 2002.

[94] A. Touzik and H. Hermann, "Theoretical study of hydrogen adsorption on graphitic materials," Chemical Physics Letters, vol. 416, no. 1-3, pp. 137-141, 2005.

[95] M. Maurer, L. Zhao, and E. Lugscheider, "Surface refinement of metal foams," Advanced Engineering Materials, vol. 4, no. 10, pp. 791-797, 2002.

[96] J. Liu, X. Zhu, Z. Huang, S. Yu, and X. Yang, "Characterization and property of microarc oxidation coatings on open-cell aluminum foams," Journal of Coatings Technology and Research, vol. 9, no. 3, pp. 357-363, 2012.

[97] J. Banhart, "Manufacture, characterisation and application of cellular metals and metal foams," Progress in Materials Science, vol. 46, no. 6, pp. 559-632, 2001.

[98] M. Ritala, M. Kemell, M. Lautala, A. Niskanen, M. Leskelä, and S. Lindfors, "Rapid coating of through-porous substrates by atomic layer deposition," Chemical Vapor Deposition, vol. 12, no. 11, pp. 655-658, 2006.

[99] J. W. Elam, G. Xiong, C. Y. Han et al., "Atomic layer deposition for the conformal coating of nanoporous materials," Journal of Nanomaterials, vol. 2006, Article ID 64501, 5 pages, 2006.

[100] J. W. Elam, J. A. Libera, M. J. Pellin, A. V. Zinovev, J. P. Greene, and J. A. Nolen, "Atomic layer deposition of W on nanoporous carbon aerogels," Applied Physics Letters, vol. 89, no. 5, Article ID 053124, 3 pages, 2006.

[101] F. Li, Y. Yang, Y. Fan, W. Xing, and Y. Ang, "Modification of ceramic membranes for pore structure tailoring: the atomic layer deposition route," Journal of Membrane Science, vol. 297298, pp. 17-23, 2012. 

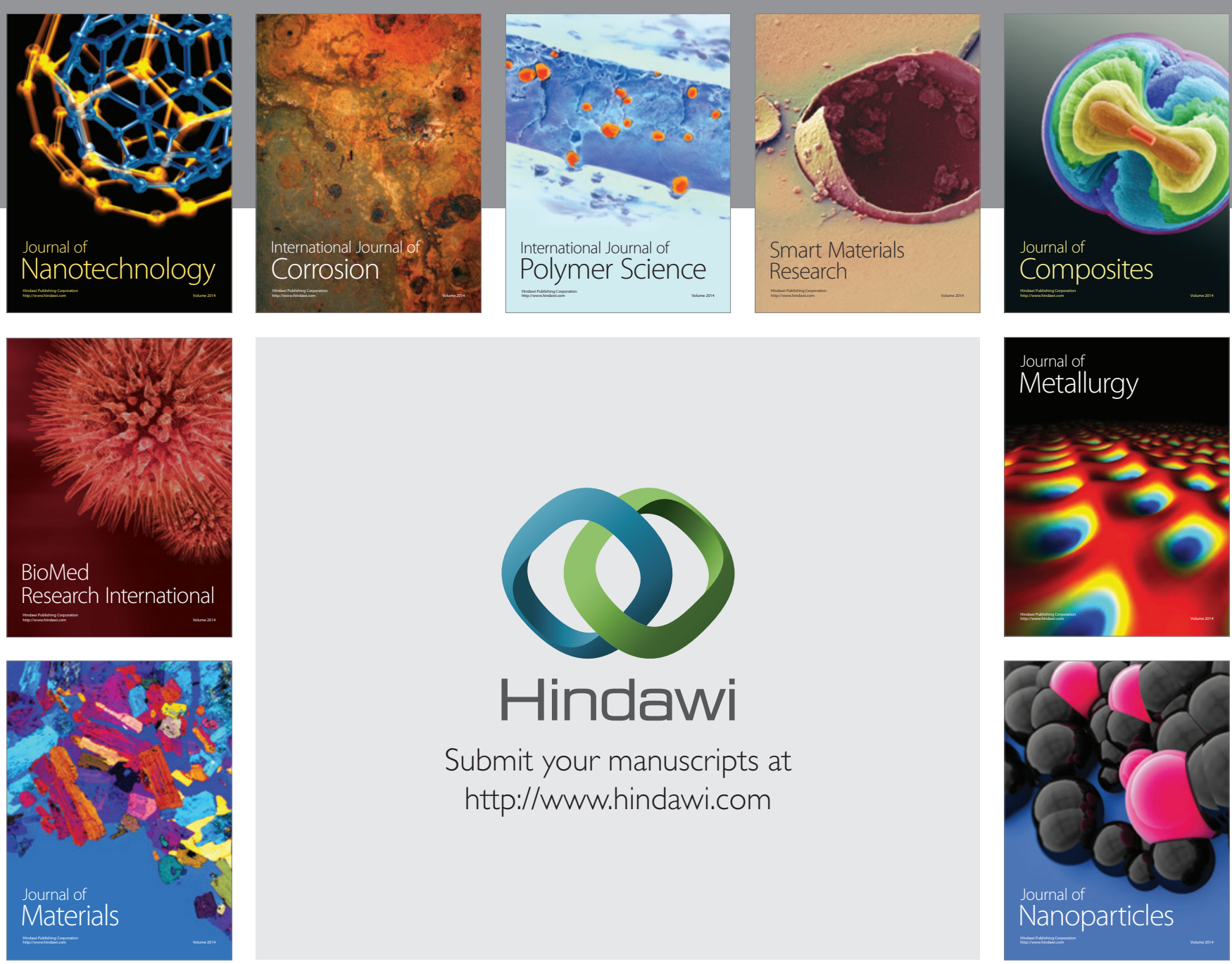

Submit your manuscripts at http://www.hindawi.com
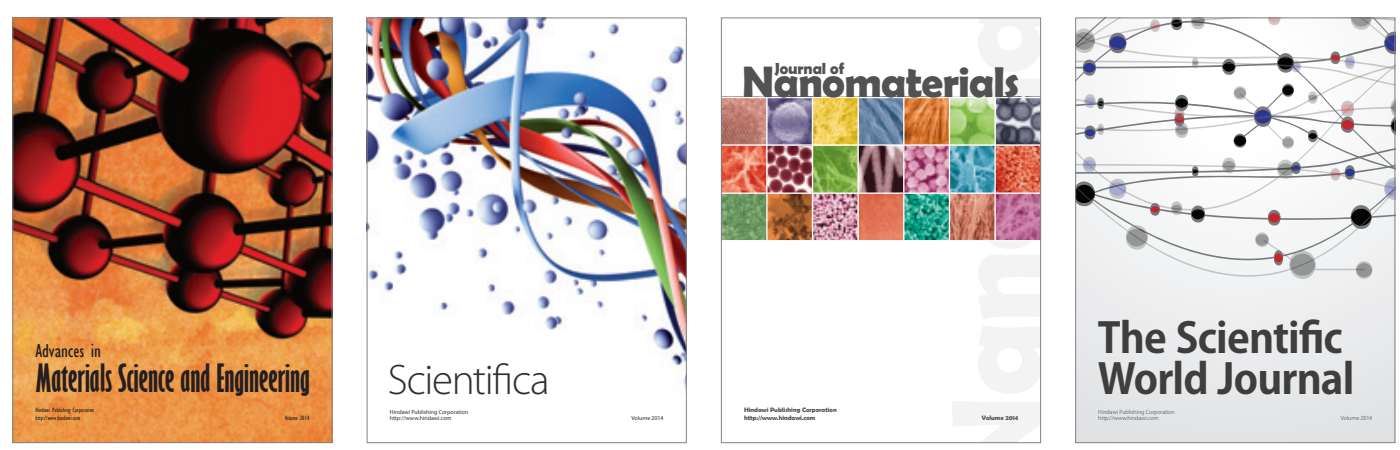

\section{The Scientific World Journal}
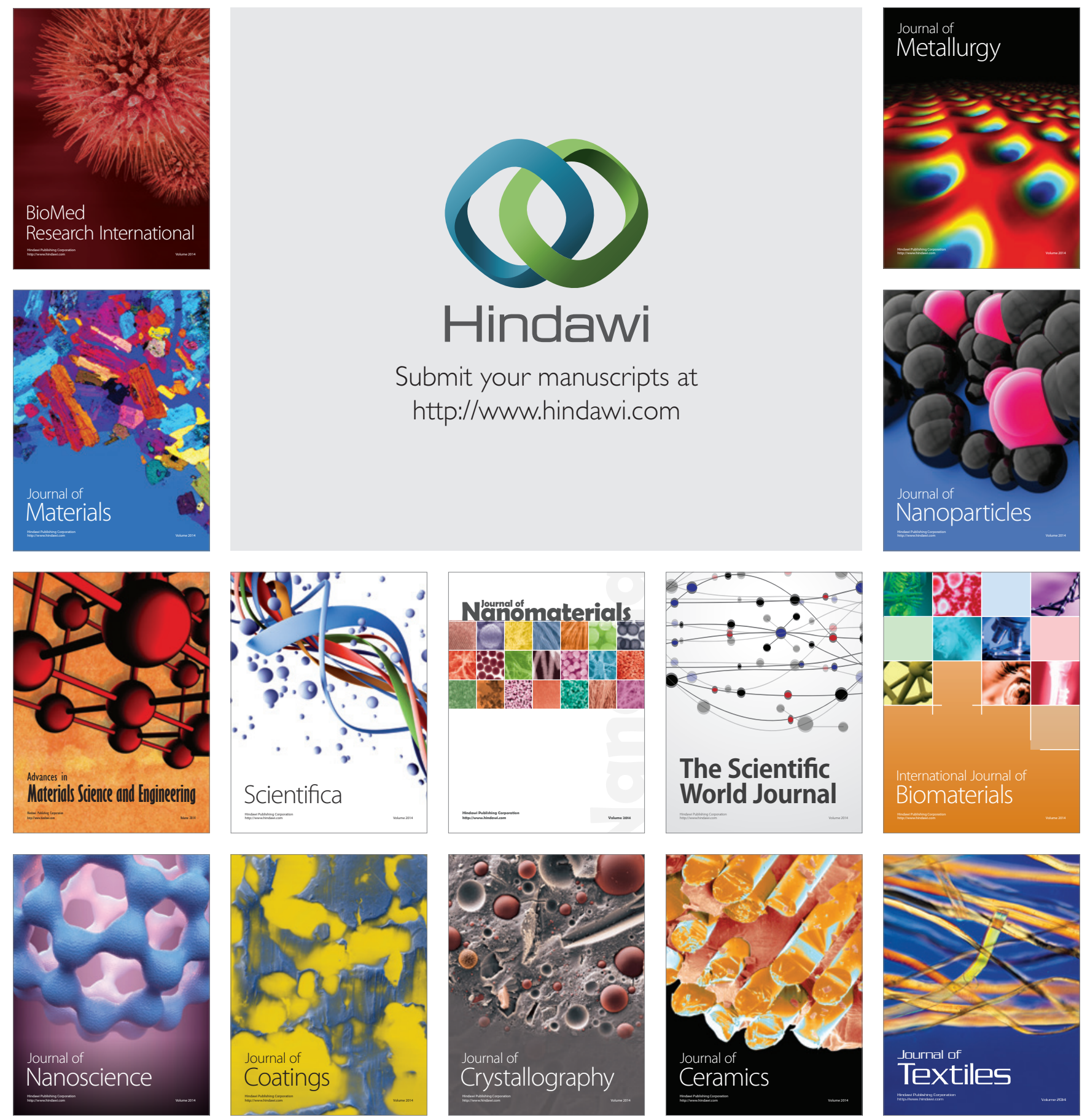